\title{
Beampattern analysis of frequency diverse array radar: a review
}

\author{
Zeeshan Ahmad, Meng Chen and Shu-Di Bao*
}

\author{
${ }^{*}$ Correspondence: \\ shudi.bao@nbut.edu.cn \\ School of Electronic \\ and Information \\ Engineering, Ningbo \\ University of Technology, \\ Ningbo 315211, People's \\ Republic of China
}

\begin{abstract}
Electronic beam steering is an essential feature of state-of-the-art radar systems. Conventional phased array (PA) radars with fixed carrier frequencies are well-known for electronically steering their beam with high directivity. However, the resulting beampattern is angle-dependent but range-independent. Recently, a new electronic beam steering concept, referred to as frequency diverse array (FDA) radar, has attracted increasing attention due to its unique range-angle dependent beampattern. More importantly, the FDA radar employs a small frequency increment across the array elements to achieve beam steering as a function of angle, range, and time. In this paper, we review the development of the FDA radar since its inception in 2006. Since the frequency offset attaches great importance in FDAs to determine the beampattern shape, initially much of the research and development were focused on designing the optimal frequency offsets for improved beampattern synthesis. Specifically, we analyze characteristics of the FDA beampattern synthesis using various frequency offsets. In addition to analyzing the FDA beampattern characteristics, this study also focuses on the neglected propagation process of the transmitted signals in the early FDA literature, and discuss the time-variant perspective of FDA beampatterns. Furthermore, FDA can also play a significant role in wireless communications, owing to its potential advantages over the conventional PAs. Therefore, we highlight its potential applications in wireless communication systems. Numerical simulations are implemented to illustrate the FDA beampattern characteristics with various frequency offset functions.
\end{abstract}

Keywords: Frequency diverse array, Frequency offset, Phased array, Range-angle dependent beampattern, Time-variant beampattern

\section{Introduction}

One of the most interesting and useful pursuits in radars today is the study of electronic scanned arrays. Electronic scanned arrays have long been of great research interest in numerous applications such as radar, sonar, navigation and wireless communications [111]. A considerable amount of effort has been expended in the investigation of methods for electronic scanning of antenna systems. During World War II, the United States and Britain were actively engaged in research on array antennas [12-14]. It was not until the 1950s that the first fully electronic scanning was realized [15-17]. In the interim, several mechanical scanners were invented and used [18]. The significant advances in the 1950s and early 1960s have made the electronic scanning using array antennas feasible with

(c) The Author(s), 2021. Open Access This article is licensed under a Creative Commons Attribution 4.0 International License, which permits use, sharing, adaptation, distribution and reproduction in any medium or format, as long as you give appropriate credit to the original author(s) and the source, provide a link to the Creative Commons licence, and indicate if changes were made. The images or other third party material in this article are included in the article's Creative Commons licence, unless indicated otherwise in a credit line to the material. If material is not included in the article's Creative Commons licence and your intended use is not permitted by statutory regulation or exceeds the permitted use, you will need to obtain permission directly from the copyright holder. To view a copy of this licence, visit http:// creativecommons.org/licenses/by/4.0/. 
the development of ferrite phase shifters in 1954-1955. Consequently, the development of Lincoln Laboratory's phased array (PA) radar began around 1958 [19]. Historically, electronically steerable phased arrays (ESA) are designed in two ways, the passive ESA and the active ESA [20]. The former utilizes a single transmitter and receiver, the latter utilizes multiple Transmit/Receive modules, typically one per element, to provide amplitude and phase control. Although scanning antenna arrays had been developed for various electronic communications applications, radar system was the first application in which PA was broadly used for the first time [20-22].

Conventional PA radars using fixed carrier frequencies are widely recognized for their capability to electronically steer a beam with high directivity [23, 24]. The effective beam of a PA radar can be electronically steered in the desired direction using phase-shifters across the array elements $[25,26]$. The resulting beampattern is angle-dependent but range-independent [27]. Therefore, PA radars cannot be deployed in applications involving range-dependent sources. On the other hand, the cost and complexity are other major concerns in PA radars because phase-shifters deployed at each antenna element represent a considerable portion of the overall system cost [23-27]. In addition, since the element-spacing in a PA system is usually fixed at half-a-wavelength corresponding to the carrier frequency, it is necessary to expand the aperture size by employing more sensors to achieve a more directed beam [20-27]. However, this will further increase the complexity, and cost. Antenna spacing could be increased above half-a-wavelength, however, this will come at the expense of multiple large side-lobes or grating-lobes in the real space when scanning the main beam [28]. Therefore, the array size dictates the number of elements in a PA beamformer [29]. Although substantial advances have taken place in PA technology since the early 1970's, affordability is still a major concern, and the design factors such as aperture sizing, pattern synthesis, and beam-switching speeds have changed little [27].

To overcome these disadvantages, and emphasizing aspects of simplicity, reliability, and versatility required by modern systems, it is necessary to find more innovative array beam steering techniques [27]. Consequently, more attention is paid to new concepts for electronic scanning of antenna systems. New concepts for electronic beam scanning proposed in this connection include waveform diversity techniques i.e., to vary the excitation across the array in either the time or frequency domain. These new configurations regarded as time-modulated arrays [30-33] and frequency scanning arrays [34, 35] can provide beam steering ability without using the expensive phase-shifters. In timemodulated arrays, high speed RF switches are used instead of expensive phase shifters to electronically steer and shape the radiation pattern [36]. The RF switches periodically turned on and off the excitations of the individual array elements according to pre-fixed time sequences [37]. In frequency scanned arrays, beam steering is achieved by the variation of frequency as a function of time, and the same signal is simultaneously applied to all the spatial channels. However, the resulted beampatterns of these techniques are still range-independent, which limit their applicability in applications involving rangedependent sources, e.g., range-dependent interference suppression, and localization of multiple targets with the same direction but distinct ranges, etc.

Recently, a new concept, namely, frequency diverse array (FDA) radar has emerged as a popular beam steering technique that generates a range-angle dependent beampattern 
[38]. The essential difference between a PA radar and an FDA radar is the carrier frequency of each array element. In contrast to PA radars using fixed carrier frequencies, FDA radars employ a small frequency increment across each array element to achieve beam steering in both the angle and range domains $[39,40]$. The concept of FDA radar was first proposed by Antonik et. al in [41], where a progressive incremental frequency shift is applied to the array signals. This progressive frequency shift generates a natural time-dependent progressive phase difference across the array elements, which enables the FDA beampattern to scan in range and angle domains as a function of time [42]. Consequently, the expensive phase-shifters are dispensed in FDA radars to steer their beam in a particular direction. The FDA radar [43] has attracted noticeable attention because the range-angle dependent beampattern is attractive to various real-time array signal processing applications including radar, sonar, wireless communications and acoustics [44-46].

FDA radar is different from orthogonal frequency division multiplexing (OFDM) radar [47-49] and multiple-input multiple-output (MIMO) radar [50-52]. OFDM radar uses orthogonal subcarriers, but non-orthogonal carriers are employed in FDA radar. MIMO radars either emit unique and independent waveforms using multiple antennas to achieve waveform diversity (i.e., collocated MIMO) or employs orthogonal signals from widely separated antennas (i.e., widely spaced MIMO) over multiple independent paths to provide spatial diversity, whereas FDA radar transmits overlapping signals with closely spaced frequencies to provide additional functionalities, such as range-dependent beamforming, increased range resolution and degree-of-freedoms (DOFs), effective mitigation of range-angle dependent interference sources and clutter. Likewise, FDA radar is also different from conventional frequency scanning radar [53, 54]. Conventional frequency scanning radar using frequency offsets as a function of time has the same frequency at each element in a given time, but FDA frequency offsets are characterized by the element index. The time-modulated array antenna [55, 56] proposed by H. E. Shanks in 1962 is a new technique for electronic scanning, which weights each element using on/off switching operation. It is noteworthy that the synthetic antenna and impulse radar (RIAS) pioneered by French researchers in early 1970's [57-59], and sparse-array synthetic antenna and impulse radar (SIAR) [60, 61] developed by Chinese researchers in 1980's are typical kinds of MIMO radar even before the concept of MIMO radar was put forward. They exploit both spatial and spectral diversity to achieve more degrees of freedom, higher angular resolution and target detection probability. MIMO radar is mainly adopted from the idea of SIAR. RIAS and SIAR simultaneously transmits a unique pulse - orthogonally phased, frequency modulated and coded and receive echoes via multiple receive antennas [62]. The concept of RIAS and SIAR also paved a way for the development of the FDA radar.

Conventional PA radar is well developed in the literature for electronically steering the beam direction with high directivity. However, the range-dependent sources cannot be unambiguously estimated from its inherent range-independent beampattern. Although space-frequency or space-time adaptive processing (STAP) based techniques can estimate the range of sources, they usually have a high computational cost and complexity [63-66]. FDA radar looks particularly promising technique for the electronic beam steering. Reference [44] overviews the basic FDA system architecture in radar and navigation applications, 
and its potential applications in range-dependent energy control and technical challenges in system implementation are discussed in [45]. Since then, there have been a number of recent advances in FDA beampattern synthesis techniques. With the increasing importance of range-angle dependent beampattern, and recent advances in FDAs, a review incorporating more recent findings has become necessary. This paper aims to briefly analyze the range-angle dependent beampattern of the FDA radar from its origin to the current stateof-the-art FDA beampatterns. While the range-angle dependent beampatterns achieved in FDAs are encouraging, the realism of the simulations is limited. More attention has been paid to achieve range-dependent beampatterns, which neglects the practical constraint of wave propagation. Therefore, we have dedicated a section to discuss the wave propagation in FDAs. In addition, we also discuss the potential applications of FDAs in wireless communications.

The outline of this paper is organized as follows. In Sect. 2, we present the PA signal model and beampattern synthesis. In Sect. 3, we describe the preliminaries of the FDA, including signal model and the time-invariant FDA beampattern synthesis. In Sect. 4, we explain the neglected propagation process of the transmitted signals in FDAs. Recent trends in FDAs are discussed in Sect. 5, and the areas that are suitable for continuing research are provided in Sect. 6. Finally, Sect. 7 concludes this study.

\section{Phased arrays}

Consider the simple case of an $M$-element uniform linear array (ULA) of isotropic radiating antenna elements with half-a-wavelength inter-element spacing as shown in Fig. 1. The farfield array radiation pattern, or array factor (AF) in this case, in terms of standard spherical coordinates, can be expressed by superposition of the $M$ radiating currents as [20-23]

$$
A F^{P A}(\theta)=\sum_{m=0}^{M-1} w_{m} \exp \{j m(\kappa d \sin \theta)\},
$$

where $w_{m}$ is the complex weight related to the $m$-th element, $\kappa=2 \pi / \lambda$ is referred to as the wavenumber with $\lambda$ denotes the wavelength of the signal, $d$ is the spacing between adjacent antenna elements, and $\theta$ is the direction of the target.

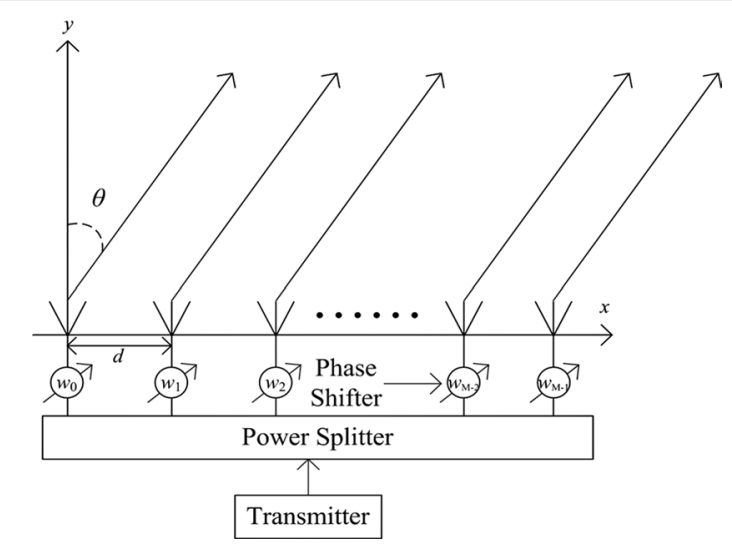

Fig. 1 Uniform linear phased array with M elements 
In what follows, the operations of transposition, complex conjugation, and Hermitian transposition are denoted by superscripts $T, *$, and $H$, respectively. Lowercase and uppercase boldface characters denote the vectors and matrices, respectively.

In vector form, Eq. (1) can be rewritten as

$$
A F^{P A}(\theta)=\boldsymbol{w}^{H} \boldsymbol{a}(\theta),
$$

where $\boldsymbol{w}$ and $\boldsymbol{a}(\theta)$, respectively, denote the weight vector and the array steering vector, which are given as [19]

$$
\boldsymbol{w}=\left[w_{0}, w_{1}, \ldots, w_{M-1}\right]^{T},
$$

and

$$
\begin{gathered}
\boldsymbol{a}(\theta)=[1, \exp (j \kappa d \sin \theta), \exp (j 2 \kappa d \sin \theta), \ldots, \\
\exp (j(M-1) \kappa d \sin \theta)]^{T} .
\end{gathered}
$$

The beam can be steered by controlling the phase shift in the weight associated with individual element. With uniform weights, i.e., $w_{m}=1$, the array factor can be expressed in closed-form as [27]

$$
\left.A F^{P A}(\theta)\right|_{w_{m}=1}=\frac{\sin ((M / 2) \kappa d \sin \theta)}{\sin ((1 / 2) \kappa d \sin \theta)} .
$$

The beampattern for the PA antenna is given as [27]

$$
B^{P A}(\theta)=\left|A F^{P A}(\theta)\right|^{2}=\left|\boldsymbol{w}^{H} \boldsymbol{a}(\theta)\right|^{2} .
$$

For the linear PA antenna to steer its main beam to angle $\theta_{d}, w_{m}$ must be equal to $\exp \left(-j m \kappa d \sin \theta_{d}\right)$, so that $B^{P A}(\theta)$ gives a maximum magnitude at that value [20-24].

$$
\begin{aligned}
B^{P A}(\theta) & =\left|\sum_{m=0}^{M-1} \exp \left\{j m\left(\kappa d\left(\sin \theta-\sin \theta_{d}\right)\right)\right\}\right|^{2}, \\
& =\left|\frac{\sin \left((M / 2) \kappa d\left(\sin \theta-\sin \theta_{d}\right)\right)}{\sin \left((1 / 2) \kappa d\left(\sin \theta-\sin \theta_{d}\right)\right)}\right|^{2}
\end{aligned}
$$

In Fig. 2, we plot the normalized beampattern of a 20-elements PA. Figure 2a, b shows the PA beampattern in range-angle dimension, and angle-dimension with the main lobe steered to $30^{\circ}$, respectively. It is observed that the PA has an angle-dependent but rangeindependent beampattern.

To further illustrate the characteristics of the PA beampattern, several array patterns with different number of elements $M$ and inter-element spacing $d$ as a function of wavelength $\lambda$ have been plotted in Fig. 3. Note that phase-shift has not been applied, and the main lobe is in the broadside direction. From Fig. 3a, it is observed that as the number of elements increases, the array directivity increases. Extending the inter-element spacing toward $\lambda$ leads to narrow main lobe but suffers high grating lobe effect as shown in Fig. 3b. The maximum grating lobe amplitude equal to the main lobe magnitude at an element spacing $\lambda$. Clearly, increasing the inter-element spacing results in a narrower main lobe but it comes at the expense of multiple large 


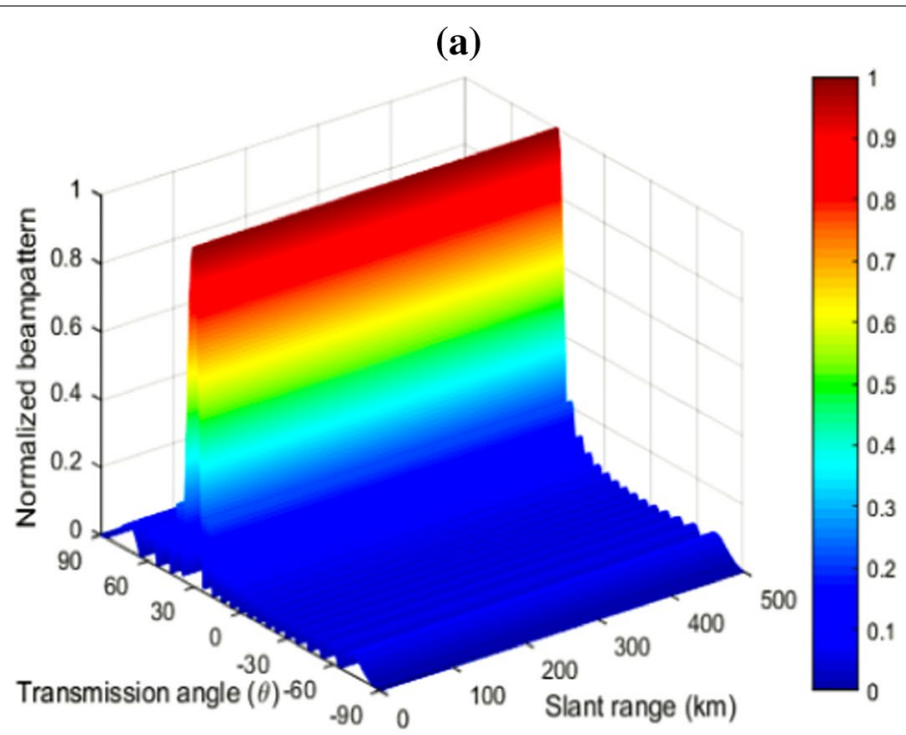

(b)

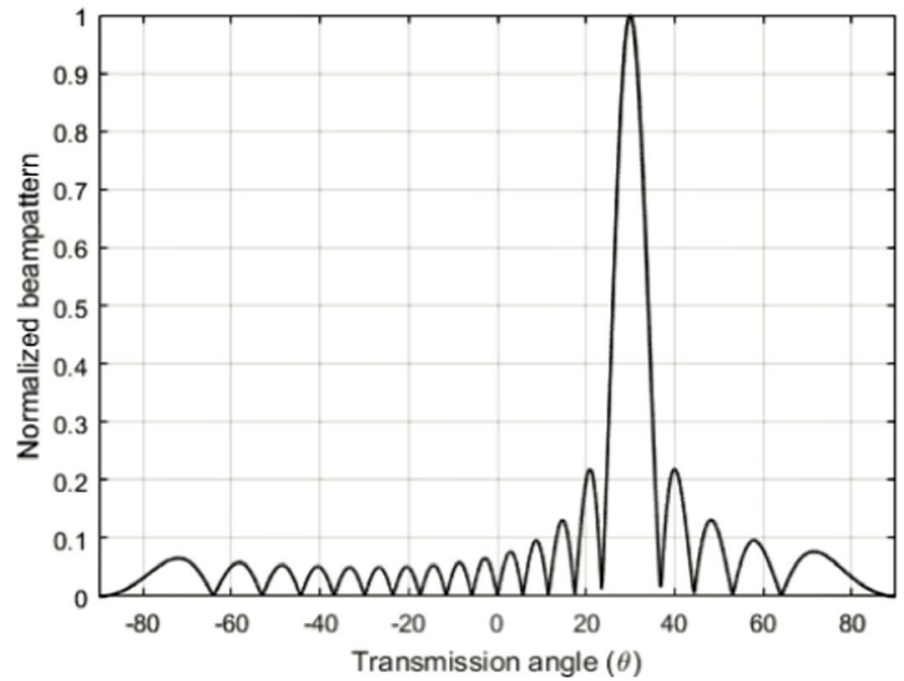

Fig. 2 Normalized beampattern of a 20-element phased array with scan angle at $30^{\circ}$. a Range-angle dimension. b Angle dimension

side-lobes or grating-lobes, whereas more antenna elements not only further decrease the main lobe width but also increase the main-to-sidelobe ratio.

\section{Frequency diverse arrays}

In this section, we briefly investigate the FDA beampattern characteristics. The first subsection formulates the signal model of FDA radars. The next subsection discusses the nonlinear frequency offsets for range-angle decoupling of the FDA beampattern. The final subsection presents the time-modulated frequency offsets to achieve the time-invariant range-angle dependent FDA beampattern. 
(a)

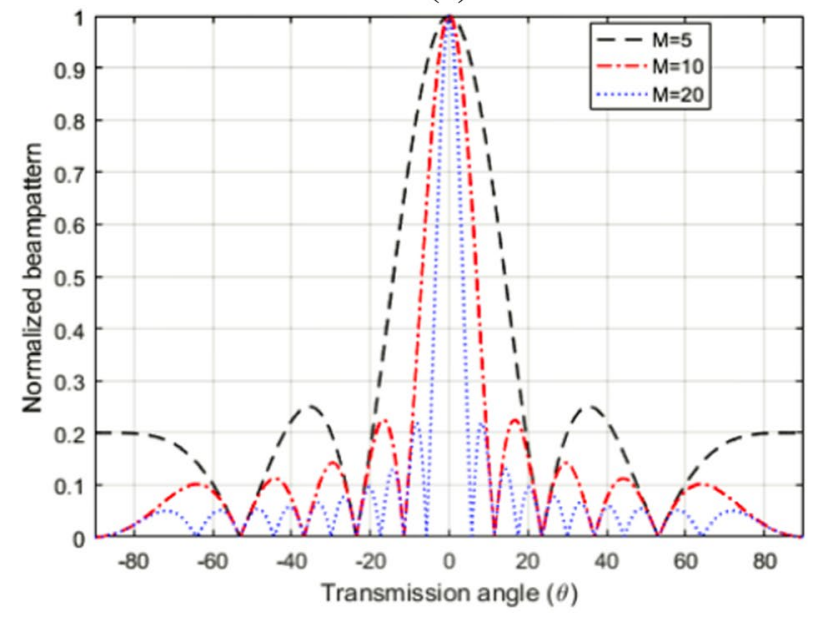

(b)

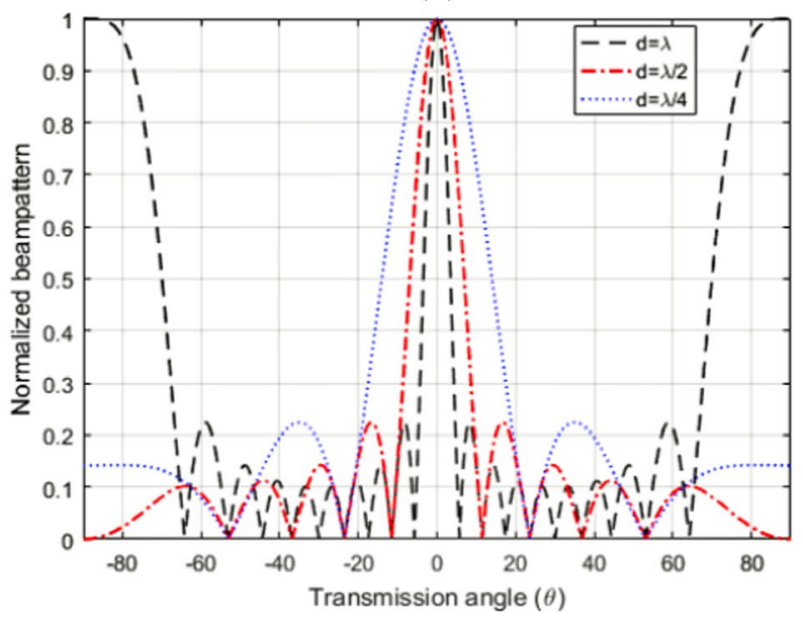

Fig. 3 Normalized phased array beampatterns with $\theta_{d}=0^{\circ}$. a Beampattern when $M=5, M=10$, and $M=15$, and $d=\frac{\lambda}{2}$. $\mathbf{b}$ Beampattern for a 10-elements phased array when $d=\lambda, d=\frac{\lambda}{2}$, and $d=\frac{\lambda}{4}$

\subsection{FDA signal model}

Assume a ULA composed of $M$ omni-directional elements with half a wavelength inter-element spacing as shown in Fig. 4. The signal transmitted by the $m$-th element is $[43-45]$

$$
s_{m}(t)=w_{m} \exp \left\{-j 2 \pi f_{m} t\right\}, \quad 0 \leq m \leq M-1, t \in[0, T]
$$

where $T$ denotes the transmitted pulse duration, and $f_{m}$ is the radiation frequency of the $m$-th element given by [38-45]

$$
f_{m}=f_{0}+m \Delta f \text {. }
$$

Here, $f_{0}$ is the carrier frequency radiated by the first element, and $m \Delta f$ is the frequency offset of the $m$-th element. The overall signal arriving at an arbitrary point $P(r, \theta)(r$ and $\theta$ are the range and the azimuth angle with respect to the first array element) in the space can be expressed as [44] 


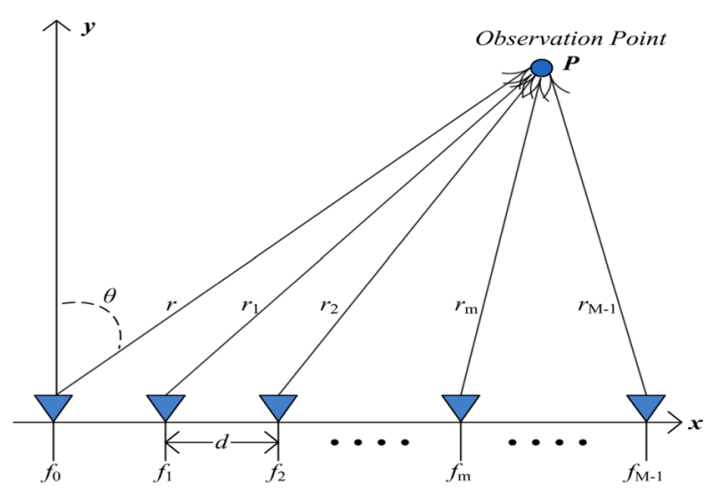

Fig. 4 Uniform linear frequency diverse array

$$
S(t, r, \theta)=\sum_{m=0}^{M-1} s_{m}\left(t-\frac{r_{m}}{c}\right)=\sum_{m=0}^{M-1} w_{m} \exp \left\{-j 2 \pi f_{m}\left(t-\frac{r_{m}}{c}\right)\right\},
$$

where $r_{m} \cong r-m d \sin \theta$ is the target slant range with respect to the $m$-th element [45], and $c$ is the wave speed. Substituting $r_{m} \cong r-m d \sin \theta$ and (9) into (10), we have [26, 45]

$$
\begin{aligned}
S(t, r, \theta)= & \sum_{m=0}^{M-1} w_{m} \exp \left\{-j 2 \pi\left(f_{0}+m \Delta f\right)\left[t-\frac{r-m d \sin \theta}{c}\right]\right\} \\
= & \exp \left\{-j 2 \pi f_{0}\left(t-\frac{r}{c}\right)\right\} \sum_{m=0}^{M-1} w_{m} \exp \{-j 2 \pi[m \Delta f \\
& \left.\left.\left(t-\frac{r}{c}\right)+m^{2} \Delta f\left(\frac{d \sin \theta}{c}\right)+f_{0}\left(\frac{m d \sin \theta}{c}\right)\right]\right\} .
\end{aligned}
$$

According to the fundamental condition of the FDA radar, the quadratic phase term $m^{2} \Delta f \frac{d \sin \theta}{c}$ satisfies the condition $m^{2}|\Delta f| \frac{d \sin \theta}{c}<\frac{\pi}{4}$ because the maximum frequency offset is far less than the carrier frequency i.e., $\max \{\Delta f\} \ll f_{0}[26,45]$. Hence, it can be ignored in the further analysis. In (11), the terms inside the summation sign are determined by the array geometry and frequency offsets of the FDA, therefore, the array factor can be expressed as [38-45]

$$
A F^{F D A}(t, r, \theta)=\sum_{m=0}^{M-1} w_{m} \exp \left\{-j 2 \pi\left[m \Delta f\left(t-\frac{r}{c}\right)+f_{0}\left(\frac{m d \sin \theta}{c}\right)\right]\right\} .
$$

Consider the case that $w_{0}=w_{1}=\ldots w_{m-1}=1$, the FDA transmit beampattern is given by 


$$
B^{F D A}(t, r, \theta)=\left|\sum_{m=0}^{M-1} \exp \left\{-j 2 \pi\left[m \Delta f\left(t-\frac{r}{c}\right)+f_{0}\left(\frac{m d \sin \theta}{c}\right)\right]\right\}\right|^{2} .
$$

The beampattern can also be expressed as $[44,45]$

$$
B^{F D A}(t, r, \theta)=\left|\frac{\sin \left[M \pi\left(\Delta f t+f_{0} d \sin \theta / c-\Delta f r / c\right)\right]}{\sin \left[\pi\left(\Delta f t+f_{0} d \sin \theta / c-\Delta f r / c\right)\right]}\right|^{2} .
$$

Note that when $\Delta f=0$, the beampattern becomes [45]

$$
B^{P A}(\theta)=\left|\frac{\sin \left[M \pi\left(f_{0} d \sin \theta / c\right)\right]}{\sin \left[\pi\left(f_{0} d \sin \theta / c\right)\right]}\right|^{2},
$$

which is only angle-dependent just like conventional PA radar.

For steering a target positioned at $P\left(r_{d}, \theta_{d}\right)$, the weights $w_{m}$ for the transmitted beampattern can be computed as [26]

$$
w_{m}=\exp \left\{-j 2 \pi\left[\frac{m \Delta f r_{d}}{c}-f_{0} \frac{m d \sin \theta_{d}}{c}\right]\right\} .
$$

The corresponding array factor becomes

$$
\begin{aligned}
A F^{F D A}\left(t, r_{d}, \theta_{d}\right)=\sum_{m=0}^{M-1} \exp \left\{-j 2 \pi\left[m \Delta f\left(t-\left(\frac{r-r_{d}}{c}\right)\right)\right.\right. \\
\left.\left.+f_{0}\left(\frac{m d\left(\sin \theta-\sin \theta_{d}\right)}{c}\right)\right]\right\},
\end{aligned}
$$

and its magnitude squared, known as transmit beampattern, is given as

$$
B^{F D A}\left(t, r_{d}, \theta_{d}\right)=\left|A F^{F D A}\left(t, r_{d}, \theta_{d}\right)\right|^{2} .
$$

Assume the following parameters: $M=10, f_{0}=10 \mathrm{GHz}, \Delta f=3 \mathrm{kHz}$, and $d=\frac{\lambda_{0}}{2}$. In Fig. 5, we plot the normalized transmit beampatterns of the PA, the standard FDA, and the symmetrical FDA. Note that $\Delta f=0$ is employed for the PA. From Fig. $5 \mathrm{a}$, it can be observed that the PA has an angle-dependent but range-independent beampattern. In contrast, from Fig. 5b we can see that the standard FDA with a progressive frequency increment yields a periodic range and angle coupled S-shaped beampattern. The symmetrical FDA has range-angle decoupled beampattern as shown in Fig. 5c.

In the transmit beampattern derived in Eq. (14), the maximum field can be obtained when the phase term satisfies the condition $[44,45]$

$$
\Delta f t+\frac{f_{0} d \sin \theta}{c}-\frac{\Delta f r}{c}=i, \quad i=0, \pm 1, \pm 2, \ldots .
$$

It can be observed from Eq. (19) that the maximum field depends not only on the angle but also on the range and time. Moreover, when only one parameter is fixed, there are multiple solutions for the unfixed parameters. On the other hand, when two parameters are fixed, the pattern periodicity depends on the unfixed variable.

For instance, if Eq. (19) is solved for time $t$, we have [44, 45] 

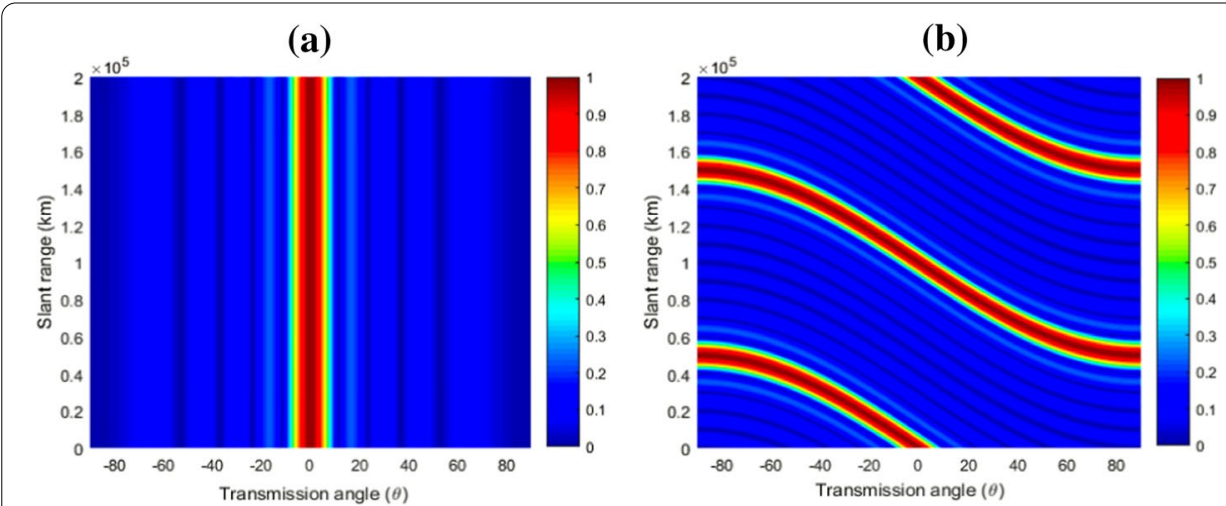

(c)

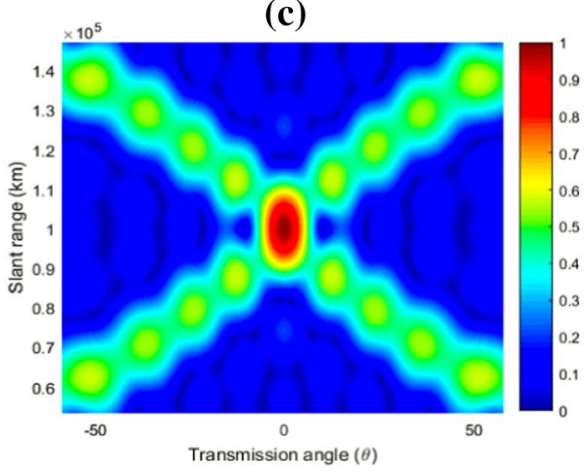

Fig. 5 Comparison of transmit beampatterns. a Phased array. b Standard FDA. c Symmetrical FDA

$$
t=\frac{i}{\Delta f}-\frac{f_{0} d \sin \theta}{c \Delta f}+\frac{r}{c}
$$

This implies the periodic nature of the FDA beampattern in time. When the range $r$ and angle $\theta$ are fixed, the fundamental period is $\frac{1}{\Delta f}$.

Similarly, the periodicity in range dimension is derived by solving Eq. (19) for range $r$ as [44]

$$
r=c t-\frac{c i}{\Delta f}-\frac{f_{0} d \sin \theta}{\Delta f},
$$

whose fundamental period is $\frac{c}{\Delta f}$ for a fixed $\theta$ and $t$.

In the same manner, if Eq. (19) is solved for $\theta$, we can obtain [45]

$$
\sin \theta=\frac{c i-\Delta f(c t-r)}{f_{0} d},
$$

Obviously, $\sin \theta$ depends on both the time and range variables. The periodicity in time, range, and angle is also shown graphically in Fig. 6 for a 17-element FDA with $10 \mathrm{kHz}$ inter-element frequency offset.

It is clear from (20)-(22) that the FDA beampattern is time-dependent, and its maxima drifts in space with the time, which facilitates the auto-scanning feature of FDA radars. That is to say, the entire space can be scanned without using the expensive phase-shifters. This unique auto-scanning feature of FDAs can also be useful 
in wireless communication systems such as at the base station and communication devices [29].

The standard FDA using progressive incremental frequency offsets generates a periodic range and angle coupled S-shaped beampattern. The range dependency of FDA beampatterns is investigated in [67-71], whereas its ability in increasing DOFs is studied in $[38,72]$. The auto-scanning property of the FDA radar is analyzed in [42, 69]. Higgins and Blunt [73] explored the range-angle coupled beamforming in FDAs, and Secmen et al. [68] described the time and angle periodicity of FDA beampatterns. In [74], Eker et al. introduced a practical FDA system using linear frequency modulated continuous waveform, wherein both the transmit and receiver architectures together with the waveform processing is being analyzed in detail. The application of the FDA for forward-looking-radar ground moving target indication, and in bistatic radar is studied in [75] and [76, 77], respectively. In [78-80], the application of FDA is extended to synthetic aperture radar for improved performance, and the frequency diversity applied to the phased-MIMO radar for range-dependent beamforming is proposed in [81]. The multipath characteristics of FDAs over a ground plane are studied in [82]. In [83], the localization performance of FDA is analyzed. Additional investigations on the two-dimensional imaging of targets and suppressing the rangedependent clutters in FDAs are, respectively, given in [84, 85] and [86, 87].

Since the range-angle coupled beampattern introduces ambiguity into target indication, the range and angle of a target cannot be unambiguously estimated using FDAs [84,

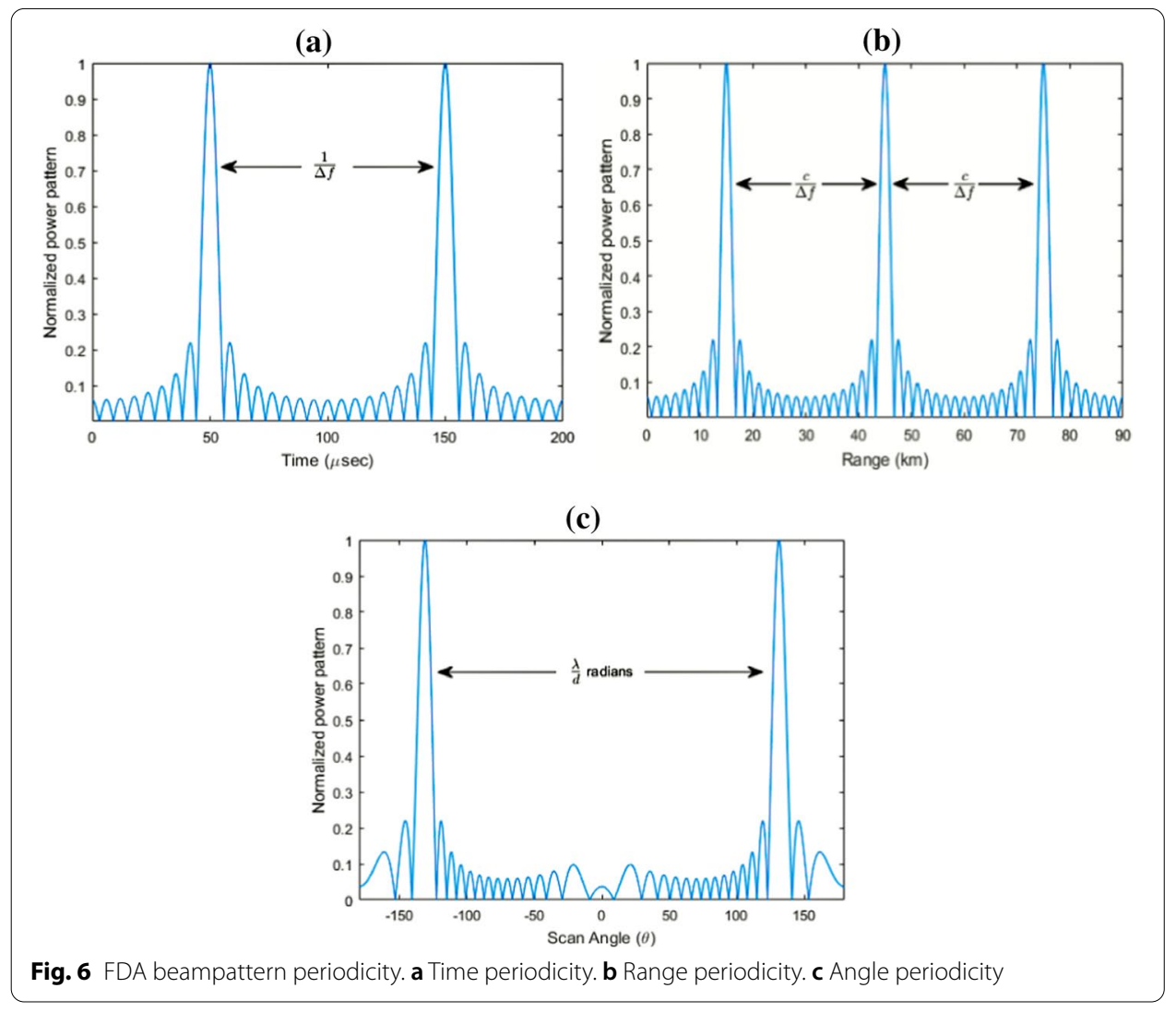


85]. There has been a long line of research in FDAs, where a series of efforts have been made to decouple its beampattern in range and angle dimensions. Among the various methods, utilizing nonlinearly increasing frequency offsets in a ULA and utilizing linearly increasing frequency offsets in nonuniform linear array are the two most representative methods to decouple the FDA beampattern in range and angle dimensions [44, 45].

Numerous other techniques were also proposed to get improved localization performance. To name a few, a double-pulse FDA radar was proposed in [88] for the rangeangle localization of targets. A pulse with zero frequency increment degenerates the system into a PA radar detecting the targets in the angle dimension, and then localize them in the range dimension using a nonzero frequency increment pulse. In [89], a stepped frequency pulse FDA (SFP-FDA) radar is proposed, which can be regarded as an upgraded version of the double-pulse FDA in [88]. The first pulse of SFP-FDA radar is same as conventional FDA with a bit of frequency increment. There is an additional small frequency increment from pulse to pulse. Another doubled pulsed MIMO-FDA has been proposed in [90], where the FDA transmit array is partitioned into subarrays and then transmits a unique waveform from each subarray with zero and nonzero frequency increment pulse, respectively. A vertical FDA, which applies frequency diversity in the vertical of a planar array, is explored in [91] to circumvent the range ambiguity problem in STAP radar. An FDA MIMO adaptive beamforming and localization scheme for range-dependent targets and interferences is proposed in [92], where the ranges and angles of targets can be solely estimated with MUSIC-based algorithm. Similarly, two FDA-MIMO hybrid radar transmitter design schemes, namely, FDA-MIMO radar, and transmit subarray FDA-MIMO radar are proposed in [93] for range-dependent target localization, where the targets are localized using the beamspace-based multiple signal classification algorithm. Although these methods have improved the localization performance, the range-angle decoupling is not achieved completely.

Considering the array configuration design, several researchers exploit the nonuniform array in FDA radars for improved localization performance. For example, a nonuniform FDA radar is proposed in [94] for range-angle imaging of targets. Likewise, a new FDA framework is proposed in [95], where the logarithmically spaced array elements were distributed symmetrically to produce a well-shaped "dot" main beam, facilitating the application in target indication. An FDA scheme utilizing nonuniform element spacing and nonlinear frequency offset is proposed in [96] to provide a range-angle decoupled beampattern with narrower main lobe and no periodicity. Moreover, a nonuniformly distributed FDA is proposed in [97], where both the frequency offsets and element positions are optimally determined via particle swarm optimization algorithm. However, it is difficult to alter the carrier frequency or frequency offsets in real time, and the relocation requirement for the accurate physical placement of transmitter and receiver at each scanning is impractical [26]. Therefore, this approach is not feasible in practice.

Another viable solution to decouple the FDA beampattern is through frequency offset design $[26,44]$. By employing nonlinear and random frequency offsets, the FDA beampattern can be efficiently decoupled into the range and angle dimensions. Therefore, the nonlinear frequency offsets are intensively researched and extensively applied in FDA's mainly for a range and angle decoupled beampattern. 


\subsection{FDA with nonuniform frequency offset}

Frequency offset design has attracted great interests in the range-angle decoupling of FDA beampatterns. Until recently, several attractive functions have been proposed to design the nonlinear frequency offsets such as square increasing and cubic increasing frequency offsets [98], Hamming window-based nonuniform frequency offsets [99], Costas sequence modulated frequency offsets [100], piecewise trigonometric frequency offsets [101], and nonuniform logarithmic frequency offsets [102].

A multi-carrier nonlinear frequency modulation FDA scheme based on logistic map is presented in [103], which is capable to reduce the main lobe width and the sidelobe peaks simultaneously. Both single-dot and multi-dot shaped range-angle dependent beampatterns are obtained in [104] by using multi-carrier frequency offsets and convex optimization. In [105], a MIMO-log-FDA radar is proposed, where the range bins concept along with logarithmic offset in each subarray of MIMO-FDA is used to produce single maxima for multiple targets present in different range bins. A uniformly spaced linear FDA with logarithmically increasing frequency offset (log-FDA), and nonuniform but symmetric frequency offsets calculated using well known mu-law formula are, respectively, proposed in [106] and [107] to achieve a beampattern with a single maximum at the target location. Moreover, an adaptive frequency offset selection scheme for FDA radar is proposed in [108], where the frequency offset is determined at each step with an iterative algorithm. Likewise, an FDA framework with Taylor windowed frequency offsets is proposed in [109] where the adjustable tapering windows determine the optimized parameters of the window function. The relationship among ambiguity, frequency pattern and target relative location is derived in [110] to identify the unambiguous frequency patterns for two-target localization. The unambiguous target localization is achieved in [111] by combining the subarray-based FDA and full-band FDA, as the transmitter and the receiver, respectively. In [112], a gridless compressed sensingbased range-angle estimation algorithm is proposed for FDA-MIMO radar via Atomic Norm Minimization and Accelerated Proximal Gradient. By optimizing frequency offsets with a genetic algorithm, a single-dot and multi-dot shape transmit beampatterns is synthesized in [113].

For illustration purpose, few typical nonlinear frequency offset functions are briefly reviewed here, and their comparative beampatterns are also analyzed.

Since the inter-element frequency offset is not uniform, therefore the frequency input at $m$-th element is

$$
f_{m}=f_{0}+\Delta f_{m}, \quad 0 \leq m \leq M-1
$$

where $\Delta f_{m}$ is the frequency offset of the $m$-th element.

The Hamming window based frequency offsets can be expressed as [99]

$$
\Delta f_{m}=\delta\left\{0.54-0.46 \cos \left(\frac{2 \pi m}{M}\right)\right\} .
$$

This is the general Hamming window equation where $\delta$ is an adjustable parameter.

The logarithmically increasing frequency offsets are computed as [106] 


$$
\Delta f_{m}=\log (m+1) \delta .
$$

The piecewise frequency offsets based on a simple trigonometric are given as [101]

$$
\Delta f_{m}=h(m) \delta
$$

where

$$
h(m)= \begin{cases}\cos \left(2 M m+\phi_{1}\right), & -M_{s} \leq m \leq 0 \\ \cos \left(M m+\phi_{2}\right), & 1 \leq m \leq M_{s}\end{cases}
$$

Here, $\phi_{1}=0$, and $\phi_{2}=\frac{\pi}{2}$. Since a symmetrical array configuration is utilized here, therefore $M=2 M_{s}+1$.

The square increasing and cubic increasing frequency offsets are derived as [98]

$$
\Delta f_{m}=\left\{\begin{array}{l}
m^{2} \Delta f \\
m^{3} \Delta f
\end{array}\right.
$$

Graphical representations of the aforementioned frequency offset functions are shown in Fig. 7.

In Fig. 8, we compare the FDA transmit beampatterns generated using the Hamming window based frequency offsets, logarithmically increasing frequency offsets, and piecewise frequency offsets based on a simple trigonometric. In this experiment, the simulation parameters are as follows: $M=17, f_{0}=10 \mathrm{GHz}, \delta=2 \mathrm{kHz}, d=\frac{\lambda_{0}}{2}$, and target location, $\left(r_{d}, \theta_{d}\right)=\left(500 \mathrm{~km}, 30^{\circ}\right)$. The results indicate that all the three FDAs achieve a focused range-angle decoupled beampattern with a single maximum pointing at the target location.

These nonlinear frequency offsets can decouple the FDA beampattern into range and angle dimensions. However, the actual beampatterns of aforementioned FDAs are timevariant. They are range-angle dependent only for a fixed time, neglecting the influence of time. Nevertheless, the performance degradation is inevitable because the time-periodicity still exists.

\subsection{FDA with time-modulated frequency offsets}

In order to overcome the time-periodicity in FDA beampatterns, a number of improved methods have been proposed [114-121]. Among those are the timedependent frequency offsets (TDFO) proposed by Khan and Qureshi [114] and a pulsed-FDA with constraints on both pulse duration and frequency shift proposed by Xu et.al. [115]. Instead of using fixed frequency offsets, the TDFO-FDA considers time-modulated frequency offsets to generate a range-angle dependent as well as time-independent beampattern. The pulsed-FDA is proposed to form a quasi-static range-angle-dependent beampattern by properly choosing the pulse duration and the frequency shift. However, the beampatterns achieved in $[114,115]$ are time-independent only for a particular range-angle pair but they remain time-dependent for other ranges and angles. To address this issue, a time-modulated optimized frequency offset FDA (TMOFO-FDA) has been proposed in [116], which exploits the combination of time-modulated and nonlinear distributed frequency offset across the array 
(a)

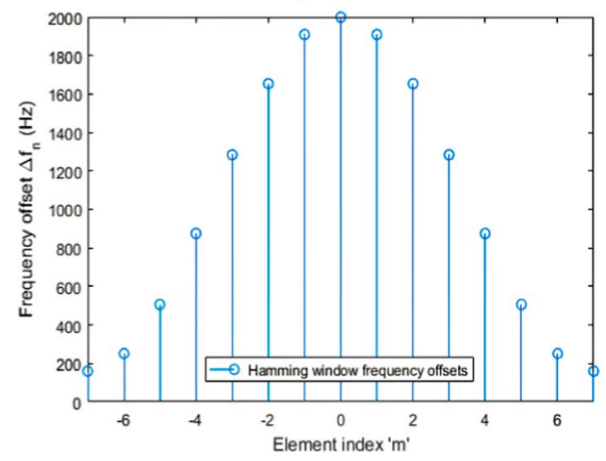

(c)

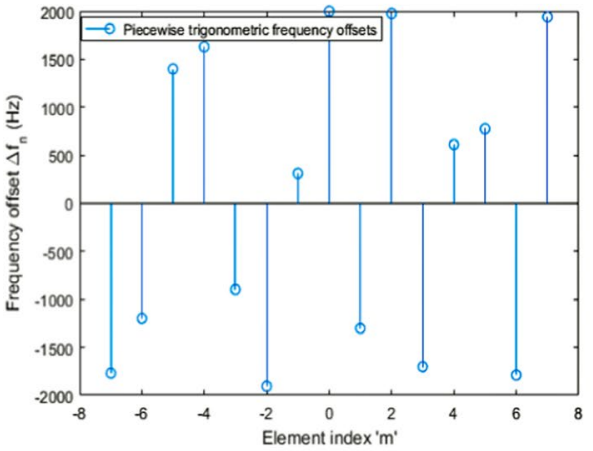

(b)

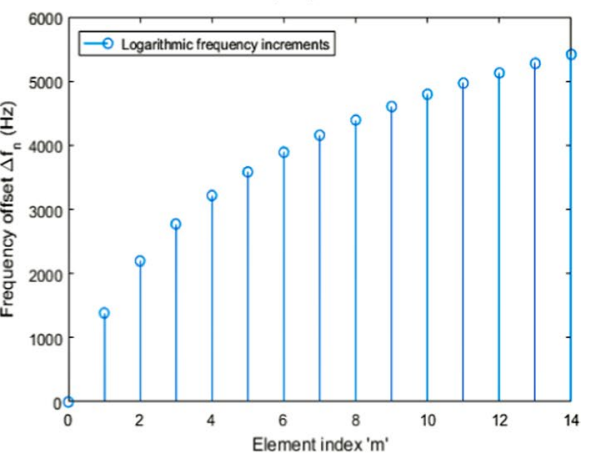

(d)

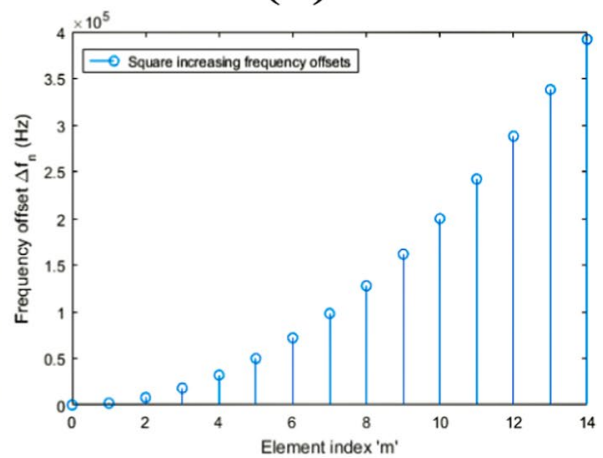

(e)

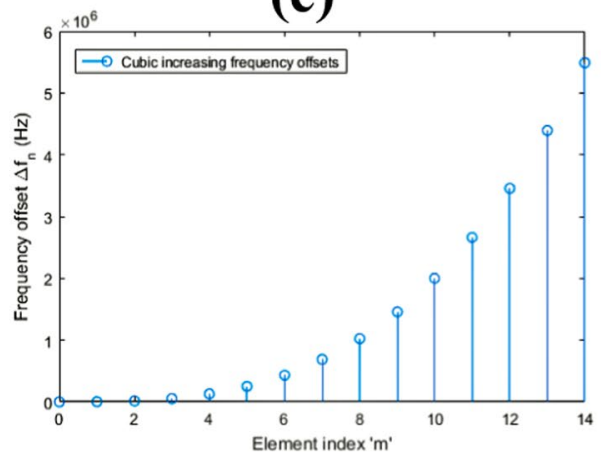

Fig. 7 Frequency offsets comparison. a Hamming window based frequency offsets. b Logarithmically increasing frequency offsets. c Piecewise trigonometric frequency offsets. $\mathbf{d}$ Square increasing frequency offsets. e Cubic increasing frequency offsets

elements to obtain a time-invariant range-angle-decoupled beampattern. By now, various improved methods based on the unified configuration of nonlinear and timemodulated frequency offsets have been proposed in [117-121] to design time-invariant spatial focusing beampatterns.

In general, the time-varying frequency offsets are given as 

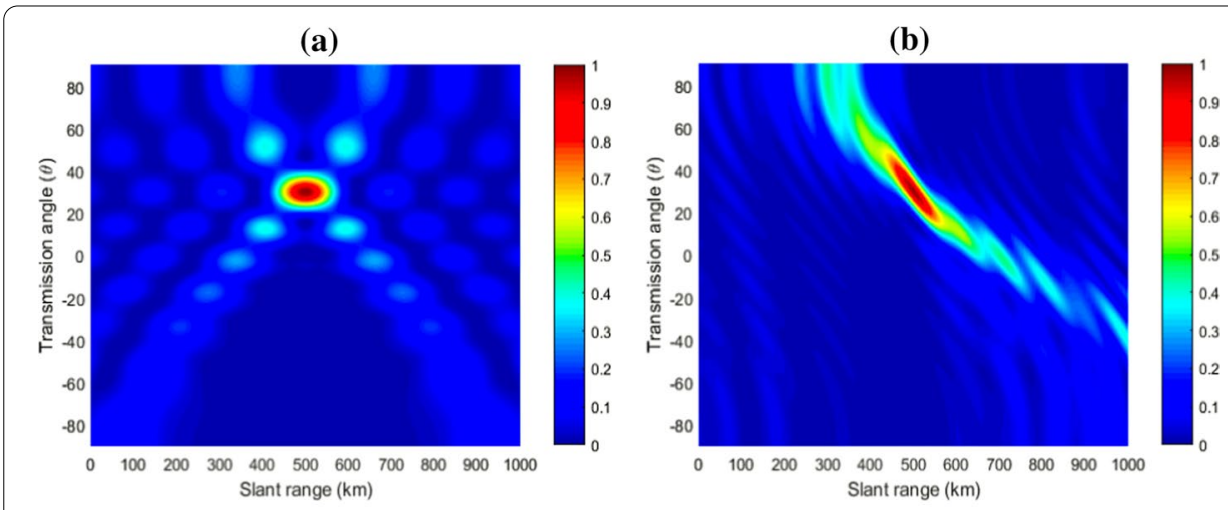

(c)

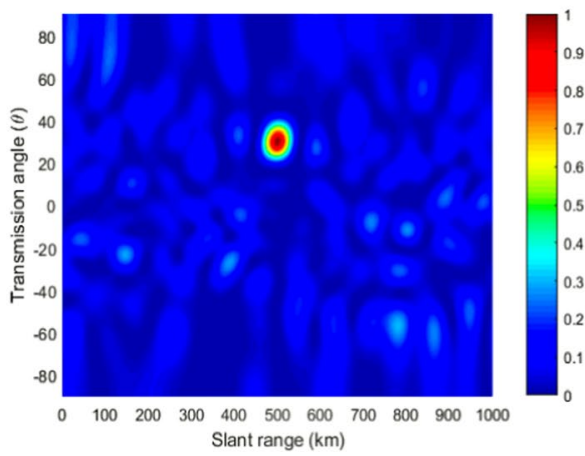

Fig. 8 Beampattern synthesis results. a Hamming FDA. b Log-FDA. c Piecewise FDA

$$
\Delta f_{m}(t)=\frac{\Delta f(m)}{t-\frac{r_{d}}{c}}, \quad t \in[0, T]
$$

where $\Delta f(m)$ is a nonlinear function with respect to the element index $m$.

As an example, we review here the TDFO-FDA [114], time-modulated logarithmically increasing frequency offset FDA (TMLFO-FDA) [116], and the time-modulated doubleparameter FDA (TMDP-FDA) [120].

The TDFO applied to the $m$ - th element is [114]

$$
\Delta f_{m}^{T D F O}(t)=m \Delta f(t)=m \frac{1-\frac{f_{0} \sin \theta_{d}}{c}}{t-\frac{r_{d}}{c}},
$$

and the array factor at the target point is expressed as

$$
\begin{aligned}
A F^{T D F O}\left(t, r_{d}, \theta_{d}\right)=\sum_{m=0}^{M-1} \exp \left\{j 2 \pi m \left[\frac{\left(t-\frac{r}{c}\right)}{\left(t-\frac{r_{d}}{c}\right)}(1-\right.\right. \\
\left.\left.\left.\frac{f_{0} d \sin \theta_{d}}{c}\right)+\frac{f_{0} d \sin \theta}{c}\right]\right\} .
\end{aligned}
$$

The TMLFO applied to the $m-t h$ element is given as [116] 


$$
\Delta f_{m}^{T M L F O}(t)=\frac{g(m)-\frac{f_{0} \sin \theta_{d}}{c}}{t-\frac{r_{d}}{c}},
$$

where $g(m)$ is a logarithmic function defined as

$$
g(m)=\ln (m+1)^{k}
$$

where $k$ is a control parameter for the frequency offset. The array factor at the target point is then expressed as

$$
\begin{aligned}
& A F^{T M L F O}\left(t, r_{d}, \theta_{d}\right)=\sum_{m=0}^{M-1} \exp \{ j 2 \pi\left[\ln (m+1)^{k}\left(\frac{t-\frac{r}{c}}{t-\frac{r_{d}}{c}}-1\right)\right. \\
&\left.\left.+\left(m \frac{f_{0} d}{c}\right)\left(\sin \theta-\sin \theta_{d} \frac{t-\frac{r}{c}}{t-\frac{r_{d}}{c}}\right)\right]\right\} .
\end{aligned}
$$

The time-modulated logistic map based frequency offsets and chirpiness constant considered in the TMDP-FDA are defined as [120]

$$
\left\{\begin{array}{l}
\Delta f_{m, n}^{T M D P}(t)=\Delta f \frac{p_{m, n}}{t-\frac{r_{d}}{c}}, \\
u_{m, n}^{T M D P}=\Delta u \frac{2 q_{m, n}}{\left(t-\frac{r_{d}}{c}\right)^{2}},
\end{array}\right.
$$

where $0 \leq n \leq N-1$; Here $\Delta f$ and $\Delta u$ are the control coefficients for the frequency offsets and chirpiness constant, respectively. It must be remembered that TMDP-FDA utilizes multi-carrier architecture, and frequency diverse chirp signal. The parameters $p_{m, n}$ and $q_{m, n}$ are generated by logistic map as [120]

$$
\left\{\begin{array}{l}
p_{m, n}=4 p_{m, n-1}\left(1-p_{m, n-1}\right), \quad 0<p_{m, 0}<1 \\
q_{m, n}=4 q_{m, n-1}\left(1-q_{m, n-1}, \quad 0<q_{m, 0}<1\right.
\end{array}\right.
$$

and the corresponding array factor at the target point is given as

$$
\begin{aligned}
A F^{T M D P}\left(t, r_{d}, \theta_{d}\right)=\sum_{m=0}^{M-1} \sum_{n=0}^{N-1} \exp \{-j 2 \pi & {\left[\frac{f_{0} m d\left(\sin \theta-\sin \theta_{d}\right)}{c}\right.} \\
& \left.\left.+\Delta f \frac{p_{m, n}\left(r-r_{d}\right)}{r_{d}}+\Delta u \frac{q_{m, n}\left(r^{2}-r_{d}^{2}\right)}{r_{d}^{2}}\right]\right\} .
\end{aligned}
$$

In this example, we assume a 10-elements ULA operating at a reference carrier frequency of $f_{0}=10 \mathrm{GHz}$. The inter-element spacing is equal to half-a-wavelength. The pulse duration is $T=1 \mathrm{~ms}$, and the target is located at $\left(r_{d}, \theta_{d}\right)=\left(500 \mathrm{~km}, 0^{\circ}\right)$. Since the multi-carrier technique is adopted in the TMDP-FDA, the number of carriers considered is $N=10$. Fig. 9 provides comparisons of beampattern generated by TDFO-FDA, TMLFO-FDA and the TMDP-FDA. It is shown in Fig. 9a that the time-independent beampattern of TDFO-FDA is periodic in range, and coupled in the range and angle dimensions. The periodicity, and range-angle coupling is eliminated in the time-invariant beampattern of TMLFO-FDA as depicted in Fig. 9b. Although the TMLFO-FDA generates a single maximum beampattern, its spatial focusing performance is degraded 

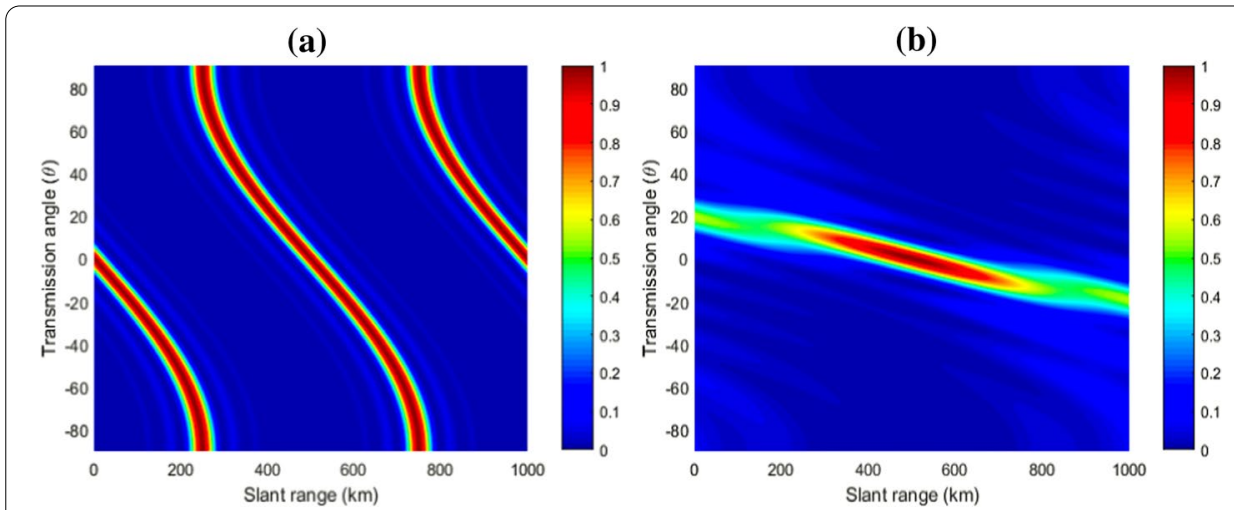

(c)

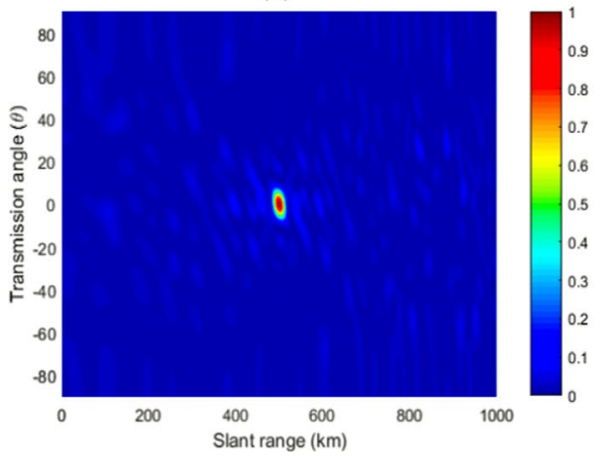

Fig. 9 Beampattern synthesis results. a TDFO-FDA. b TMLFO-FDA. c TMDP-FDA

due to the high spatial peak sidelobe levels (P-SLL) and a broader spatial half-power beamwidth (HPBW). Furthermore, as Fig. 9c shows, the TMDP-FDA generate a wellshaped "dot" main beam and outperforms the other two schemes with a more focused beampattern due to multi-carrier architecture.

To further demonstrate the resolution and side-lobe suppression performance, the corresponding range, and angle dimension beampatterns are plotted in Fig. 10. From Fig. 10a, it can be found that all the three FDAs have equal response in angle dimension except that the TDFO-FDA beampattern exhibit periodicity. However, from Fig. 10b, it is observed that the TMDP-FDA outperforms the TDFO-FDA and TMLFO-FDA with narrow $\mathrm{HPBW}$ in range dimension. The TDFO-beampattern exhibit periodicity in range dimension, and the performance of TMLFO-FDA is not satisfactory due to the high P-SLL and the broad spatial HPBW. Instead of a single carrier signal, each array element of the multi-carrier TMDP-FDA transmit a weighted summation of signals with a small frequency offset. Therefore, the effect of multi-carrier architecture on the performance of the TMDP-FDA is also demonstrated in Fig. 10c. It is observed that as the number of carrier frequencies increases, the P-SLL suppression and the HPBW gets improved.

Although much attention has been paid to the uniform linear array configurations in existing FDAs due to its simple structure and well-developed techniques, other array configurations are also very appealing for non-communication applications such as radar, localization and positioning [122]. Numerous related works exploring FDAs with different array configurations for improved performance can be found in [123-129]. 

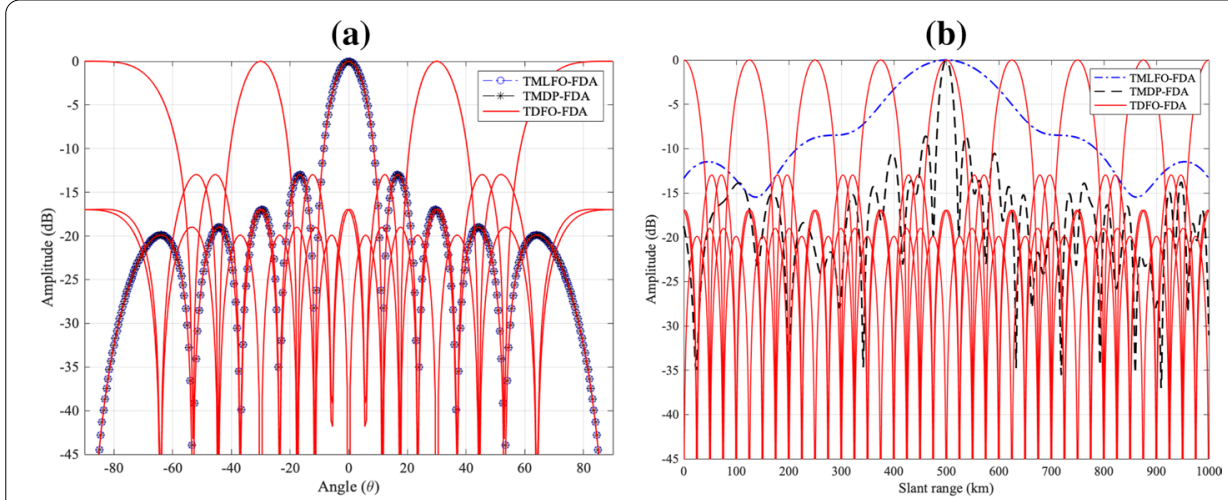

(c)

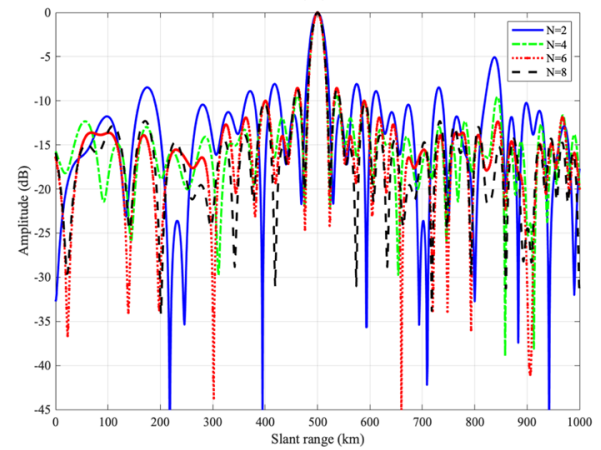

Fig. 10 Comparison of beampatterns in a angle dimension. $\mathbf{b}$ range dimension. c TMDP-FDA with varying numbers of carrier frequencies

For instance, a multitarget localization algorithm for the sparse-FDA radar is proposed in [123], which incorporates both coprime arrays and coprime frequency offsets. Likewise, the compressive sensing technique is applied to FDA in [124] for range-angle estimation with a new type of array antenna, named random frequency diverse array. In [125], a joint optimization design scheme for FDA-MIMO radar is proposed, where virtual coprime planar array with 'unfolded' coprime frequency offsets framework is utilized to achieve three-dimensional (3D) localization without ambiguity. Similarly, a new waveform synthesis model of time modulation and range compensation FDA-MIMO is proposed in [126] to achieve a joint range-angle estimation based on the optimized timeinvariant and dot-shaped beampattern. The beampattern analysis in terms 3D beam steering and auto scanning capabilities of the uniform circular FDA (UC-FDA) and the planar FDA were discussed in [127, 128] and [129], respectively. A flat-top range-angle dependent beampattern synthesis method has been proposed in [130] for FDA based on second-order cone programming. Similarly, a subarray-based FDA framework is devised in [131] to achieve a range-angle decoupled beampattern. A circular FDA utilizing tangent hyperbolic function for frequency offsets selection scheme is proposed in [132], where three different configurations of an FDA can be generated by adjusting a single function parameter of the tangent hyperbolic function.

The aforementioned FDA techniques can obtain a time-invariant range-angle decoupled beampattern, however, some recent studies indicate that FDA beampatterns are 
always time-variant in free space [133]. The existing FDA techniques designed for the range-angle dependent beampattern synthesis have not considered the propagation process of the transmitted signals, and may suffer performance degradation caused by the wave-propagation [133]. Consequently, it is necessary to revisit the FDA signal model and considering the wave propagation phenomenon into account, the new signal model is devised with time-variant focusing capability.

\section{Wave propagation effect in FDAs}

\subsection{Correction of the time parameters}

The original array factor of the FDA is a function of time $t$, which indicates that the EM waves propagate at the speed of light. More importantly, the term " $t-\frac{r}{c}$ " in (12) indicates that the range $r$ and time $t$ are correlated, and their relationship cannot be ignored [133, 134]. As highlighted in [134], the propagation process of the transmitted signals is neglected in the early FDA literature, and the time-invariant beampatterns for FDAs were obtained by assuming the time variable $t$ to be a fixed value (e.g., $t=0$ ) or defining the variable $t$ in the range from 0 to $T$ [135]. This assumption that the signals propagated to the target location without time consumption is not valid in practice [133, 134].

Recall the array factor of TMLFO-FDA given by Eq. (34) as follows

$$
\begin{aligned}
& A F^{T M L F O}(t, r, \theta)=\sum_{m=0}^{M-1} \exp \{ j 2 \pi\left[\ln (m+1)^{k}\left(\frac{t-\frac{r}{c}}{t-\frac{r_{d}}{c}}-1\right)\right. \\
&\left.+\left(m \frac{f_{0} d}{c}\right)\left(\sin \theta-\sin \theta_{d} \frac{t-\frac{r}{c}}{\left.t-\frac{r_{d}}{c}\right)}\right]\right\} .
\end{aligned}
$$

In (38), when $(r, \theta)=\left(r_{d}, \theta_{d}\right)$, we have

$$
\left.A F^{T M L F O}\left(t, r_{d}, \theta_{d}\right)\right)=\sum_{m=0}^{M-1} \exp \{-j 2 \pi(0)\}=M
$$

In (38)-(39), the maximum of the range-angle-dependent beampattern stayed at the target location is independent of time as long as the range and angle are satisfied with $r=r_{d}$ and $\theta=\theta_{d}$. Furthermore, the beampattern is calculated during $t \in[0, T]$. However, considering the propagation process of the transmitted signals, it is impractical that the EM waves propagate to a certain point $P(r, \theta)$ without consuming time, i.e., at $t=0$. Similarly, it is also unconvincing to achieve the desired beampattern within the pulse duration that is at $t=T$, because the desired range is usually taken as $r_{d} \gg T$.c [135].

Similarly, in the FDA schemes proposed in [114-121], the time-modulated frequency offsets were directly substituted in (12), and the variation of $\Delta f_{m}(t)$ is supposed to propagate to any position without time consumption [134-138]. Hence, the beampatterns at the target point remain static all the time for $r=r_{d}$ and $\theta=\theta_{d}$. It is worth noticing that the range factor is introduced by the time factor in FDAs, however, the direct substitution of $\Delta f_{m}(t)$ also indicates that the range influence is ignored. In other words, the range $r$ is bounded up with time $t$, i.e., where there is $t$, there is $r$, and where there is $r$, there is $t$. 
Hence, the array factor of these FDAs should be corrected by incorporating $t-\frac{r}{c}$ as a single quantity variable of function $f$ for the signals propagating in free space. Therefore, Eq. (29) can be revised as [134-136]

$$
\Delta f_{m}(t)=\frac{\Delta f(m)}{t-\frac{r_{m}}{c}-\frac{r_{d}}{c}} .
$$

The corrected array factor of TMLFO-FDA [116] is then derived as

$$
\begin{aligned}
& A F^{T M L F O}(t, r, \theta)=\sum_{m=0}^{M-1} \exp \{ j 2 \pi\left[\ln (m+1)^{k}\left(\frac{t-\frac{r}{c}}{t-\frac{r}{c}-\frac{r_{d}}{c}}-1\right)\right. \\
&\left.+\left(m \frac{f_{0} d}{c}\right)\left(\sin \theta-\sin \theta_{d} \frac{t-\frac{r}{c}}{\left.t-\frac{r}{c}-\frac{r_{d}}{c}\right)}\right]\right\} .
\end{aligned}
$$

It can be observed from (41), that the maximum of the correct beampattern of TMLFOFDA no longer stays at the target point $\left(r_{d}, \theta_{d}\right)$. The original and correct beampattern of the TMLFO-FDA is compared in Fig. 11. From the beampatterns projected on the range-time dimension shown in Fig. 11a, b, it is observed that the original beampattern of TMLFO-FDA at the target point is time-invariant, however, when the propagation process of the transmitted signals is taken into account, the focus line disappears. The correct TMLFO-FDA beampattern is unable to focus the transmit energy at the desired target, when the practical signal propagation process is taken into account. The comparison of original and corresponding corrected models of some prominent FDAs is shown in Table 1.

The unnoticed misconceptions about the time-range relationship, and frequencyphase relationship in FDAs are briefly analyzed in [134]. Additionally, the difference between the time-index within the pulse duration $T$, and the actual time $t$ is also explained in [136]. It is shown that the variable $t$ represents the actual time of the signal traveling in the space, whereas $T$ is the time index within the pulse duration. More importantly, it is shown that the range parameter is related to the wave propagation i.e., time variable $t$, while phase of the wave signal is related to the pulse duration $T$, but not vice versa [136]. Based on the frequency-phase relationship, and the time-range relationship, these studies reestablish the correct signal models. It is proved that the beampattern of FDA is always time-variant as long as the electromagnetic waves travel at the velocity of light. It is worthy of note that existing FDA techniques mainly designed for time-invariant beampattern synthesis will essentially lead to a range-invariant beampattern as in conventional antenna arrays [137]. In other words, the design of time-invariant range-dependent beampattern is impractical. Consequently, these FDA schemes cease to focus the transmit energy at the desired target location when the wave propagation property is considered [138].

\subsection{Scope of the time variable}

Practically, $c \Delta t$ movement in range is obvious for an elapsed time $\Delta t$. For instance, the minimum time required for EM waves propagating with the speed of light to reach the target located at range $r_{d}$ is $t=\frac{r_{d}}{c}$. For a signal with pulse duration $T$, the target can be illuminated for $T$ seconds and beyond that time, there is no signal. So, the scope of the 


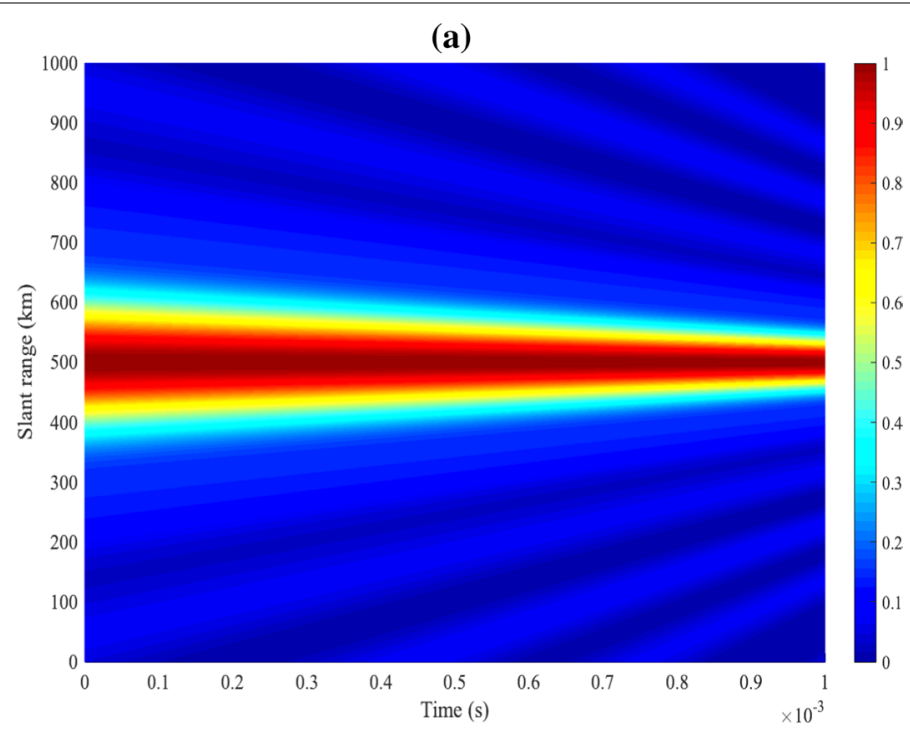

(b)

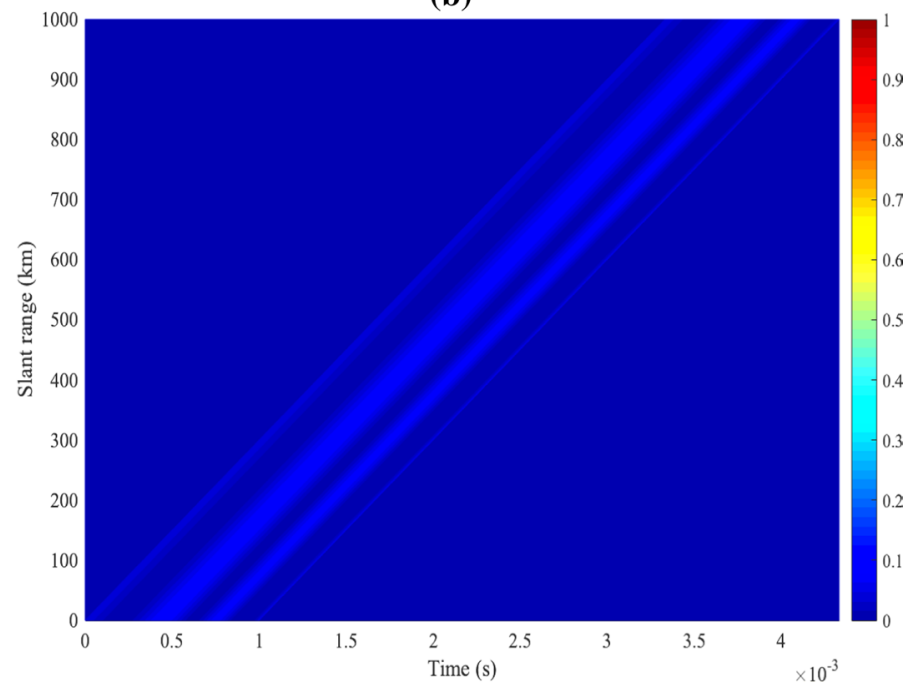

Fig. 11 Transmit beampattern of TMLFO-FDA in range-time dimensions with $\left(r_{d}, \theta_{d}\right)=\left(500 \mathrm{~km}, 0^{\circ}\right)$. a Original time-invariant beampattern. $\mathbf{b}$ Corresponding correct beampattern

Table 1 Comparison of original and corrected FDA array factors

\begin{tabular}{|c|c|c|}
\hline Type of FDA & Original array factor & Corrected array factor \\
\hline $\begin{array}{l}\text { TDFO-FDA } \\
{[114]}\end{array}$ & $\sum_{m=0}^{M-1} e^{j 2 \pi m\left[\frac{t-r / c}{t-r_{d} / c}\left(1-\frac{f_{0} d \sin \theta_{d}}{c}\right)+\frac{f_{0} d \sin \theta}{c}\right]}$ & $\sum_{m=0}^{M-1} e^{j 2 \pi m\left[\frac{t-r / c}{t-r / c-r_{d} / c}\left(1-\frac{f_{0} d \sin \theta_{d}}{c}\right)+\frac{f_{0} d \sin \theta}{c}\right]}$ \\
\hline $\begin{array}{l}\text { Pulsed-FDA } \\
{[115]}\end{array}$ & $\operatorname{rect}\left(\frac{t}{T_{p}}\right) \sum_{m=0}^{M-1} e^{j 2 \pi m\left[\Delta f\left(t-\frac{r_{m}}{c}\right)+\frac{f_{0} d \sin \theta}{c}\right]}$ & $\operatorname{rect}\left(\frac{t-r_{m} / c}{T_{p}}\right) e^{j 2 \pi f_{0}\left(t-\frac{r}{c}\right)} \sum_{m=0}^{M-1} e^{j 2 \pi m\left[\Delta f\left(t-\frac{r_{m}}{c}\right)+\frac{f_{0} d \sin \theta}{c}\right]}$ \\
\hline $\begin{array}{l}\text { C-TMOFO FDA } \\
{[119]}\end{array}$ & $\begin{array}{l}\sum_{m=-M}^{M} e^{\left\{2 \pi\left[\left(g(m)-\frac{m \sin \theta_{d}}{2}\right)\left(\frac{t-\frac{r}{\tau}+\frac{m \sin \theta}{2 t_{0}}}{t-\frac{r d}{c}+\frac{m \sin \theta_{d}}{2 f_{0}}}\right)\right.\right.} \\
\left.\left.+\frac{m \sin \theta}{2}-g(m)\right]\right\}\end{array}$ & $\sum_{m=-M}^{M} e^{\left\{2 \pi\left[\left(g(m)-\frac{m \sin \theta_{d}}{2}\right)\left(\frac{t-\frac{r}{\tau}+\frac{m \sin \theta}{2 f_{0}}}{t-\frac{r}{c}-\frac{r d}{c}+\frac{m \sin \theta_{d}}{2 f_{0}}}\right)+\frac{m \sin \theta}{2}-g(m)\right]\right\}}$ \\
\hline
\end{tabular}


time related to wave propagation for a desired target located at $P\left(r_{d}, \theta_{d}\right)$ can be revised as $t \in\left[\frac{r_{d}}{c}, \frac{r_{d}}{c}+T\right]$. The corrected array factor can then be expressed as [133]

$$
A F\left(t, r_{d}, \theta_{d}\right)=\left\{\begin{array}{lc}
\sum_{m=0}^{M-1} w_{m} \exp \left\{j \psi_{m}\right\}, & t \in\left[\frac{r_{d}}{c}, \frac{r_{d}}{c}+T\right] \\
0, & \text { otherwise }
\end{array}\right.
$$

where $\psi_{m}=-2 \pi\left[\Delta f_{m}\left(t-\frac{r}{c}\right)+\frac{f_{0} m d \sin \theta}{c}\right]$.

In summary, most signal models in time-invariant theories are actually inappropriate because the time-range relationship is ignored. The neglected propagation process of the transmitted signals causes essential misconception and results in erroneous conclusions. The term " $t-\frac{r}{c}$ " indicates that maximum of the FDA beampattern cannot be independently controlled in time and range dimension, and the focusing point always moves with the velocity of light. Nevertheless, the performance degradation in time-invariant FDAs is inevitable when the wave propagation is considered, leading to the movement of focusing point with time. However, it is important to note that constant range for the focus point of FDAs appears to be unattainable but achieving a dot-shaped beampattern with FDAs in an instant of time is not in contradiction with the physical phenomenon of EM wave propagation $[134,138]$.

\section{Recent trends in FDAs}

\subsection{Time-variant focusing FDA beampattern synthesis}

Considering the propagation process of transmitted signals in FDAs, several researchers strive to focus the transmit energy on the desired target in an instant of time. For example, a short-range symmetrical FDA radar is proposed in [135] by incorporating an arc-tangent function modulated frequency scheme. Likewise, a transmit beampattern synthesis method based on arc-tangent function is proposed in [139] for waveform diverse array radar to focus the signal energy on the desired target for a period of time. A multi-carrier symmetrical FDA radar with frequency diverse chirp signal is proposed in [140] for short-range applications. They achieve a time-variant focused range-angle dependent beampattern by compensating the propagation delays of transmitting signals. In [141], a focused angle-range beamforming synthesis method with time-varying characteristics is proposed, where the frequency offsets, amplitude weighting and phase weighting are optimized by bat metaheuristic algorithm to achieve single-dot and multi-dot shape transmit beamforming. A logarithm-based optimized static nonlinear frequency offset for FDA is proposed in [142] to alleviate its intrinsic time-variant problem, which depends on a frequency offset control function and the duration of a short-time interval rather than introducing the time variable. More recently, investigations on exploring different array configurations for improved performance have been made in [143-146]. A dual function range-angle dependent sidelobe control using FDA-MIMO is proposed in [143] for joint radarcommunications. The proposed system considers the time-variance of an FDA beampatterm. A high-speed user-centric beampattern synthesis method for FDA is devised in [144], where the time-variant beampattern peak accompany the quickly-moving user without altering the frequency offsets. A UC-FDA radar is proposed in [145] to realize time-variant spatial focusing in short-range at the target location. The UCFDA radar is capable to provide $360^{\circ}$ of coverage in the azimuth plane, which cannot 
be achieved by the linear array-based FDA radar. Similarly, a hemi-spherical FDA is exploited in [146] to demonstrate the unique advantage of conformal FDA in terms of 3D localization ability. It alleviates the inherent time-variant problem, and generate a quasi-time-invariant 3D focusing beampattern. The FDA schemes presented in [145, 146] incorporates pulse-dependent frequency offsets to achieve time-variant spatial focusing beampattern.

The array factor for the short-range FDA radar proposed in [135] is given as

$$
A F\left(t, r_{d}, \theta_{d}\right)=\sum_{m=-M}^{M} \exp \left\{-j 2 \pi \psi_{m}\left[t-\frac{r_{m}}{c}+\Delta \tau_{m}\right]\right\},
$$

where $\quad \psi_{m}(t)=\Delta f_{m} \frac{2}{\pi}\left(t-\frac{T}{2}\right) \arctan \left[\frac{\alpha}{T}\left(t-\frac{T}{2}\right)\right]-\Delta f_{m} \frac{T}{\alpha \pi} \ln \left[\frac{\alpha^{2}}{T^{2}}\left(t-\frac{T}{2}\right)^{2}+1\right], \quad$ and $\Delta \tau_{m}=\frac{r_{m}^{\prime}-r_{d}}{c}$ is the corresponding transmit delay, where $r_{m}^{\prime}=r_{d}-m d \sin \theta_{d}$.

The pulse-dependent nonlinear frequency offset proposed in [145] is defined as

$$
\Delta f_{m, n}(t)=\frac{\alpha g(m, n)-f_{0} \frac{a}{c} \sin \theta_{d} \cos \left(\varphi_{d}-\varphi_{m}\right)}{t-\frac{r_{d}}{c}+\frac{a}{c} \sin \theta_{d} \cos \left(\varphi_{d}-\varphi_{m}\right)}
$$

where $\varphi$ denotes the elevation angle, and $g(m, n)$ is a nonlinear function generated by a logistic map with a control parameter $\alpha$. Since the transmitted signals can propagate to the target location $P\left(r_{d}, \theta_{d}, \varphi_{d}\right)$ within the time interval $t \in\left[\frac{r_{d}}{c}, \frac{r_{d}}{c}+T\right]$, therefore, assuming the value of $t$ within this range, the final form of pulse-dependent frequency offsets becomes

$$
\Delta f_{m, n}(m, n)=\frac{\alpha g(m, n)-f_{0} \frac{a}{c} \sin \theta_{d} \cos \left(\varphi_{d}-\varphi_{m}\right)}{\beta T+\frac{a}{c} \sin \theta_{d} \cos \left(\varphi_{d}-\varphi_{m}\right)},
$$

where the introduced coefficient $\beta$ satisfies the condition $0 \leq \beta \leq 1$.

Similarly, the optimized static nonlinear frequency offset (OSNFO) for general conformal FDA in [146] is given by

$$
\Delta f_{m, n}^{O S N F O}=\frac{g_{O S N F O}(m)}{T / 2},
$$

where $g_{O S N F O}(m)$ is a nonlinear function to be optimized by the conventional evolutionary methodology.

In Fig. 12, we have demonstrated the significance of the wave propagation effect in FDAs. Figure 12a shows the original time-invariant beampattern generated by the compensated TMOFO (C-TMOFO) FDA [119], where a focused beampattern with a single maximum at the target position is achieved. Figure $12 \mathrm{~b}$ shows its corresponding corrected beampattern projected on the time-range dimension when wave propagation is considered. Obviously, the focused point disappears, and the beampattern is unable to be steered to the target position. The beampattern of the short-range FDA radar based on arc-tangent function [135] is shown in Fig. 12c. It is observed that the transmit energy is focused when the pulse signals reach the desired spatial region $r_{d}=6 \mathrm{~m}$ at $t=25 \mathrm{~ns}$ only. The analysis of time-invariant FDAs, and their corrected models with simulated examples are briefly discussed in [134-140, 145]. 

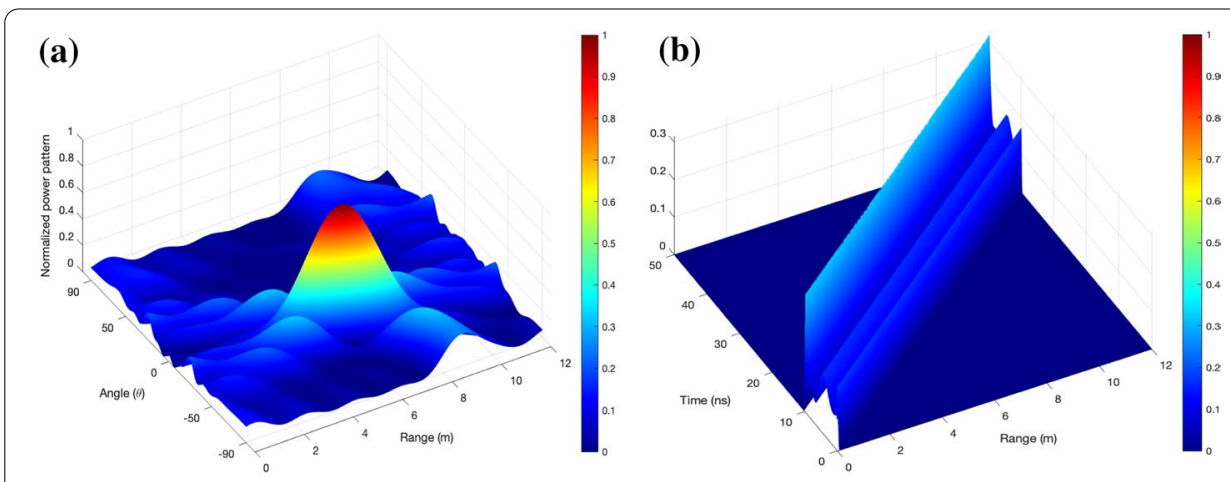

(c)

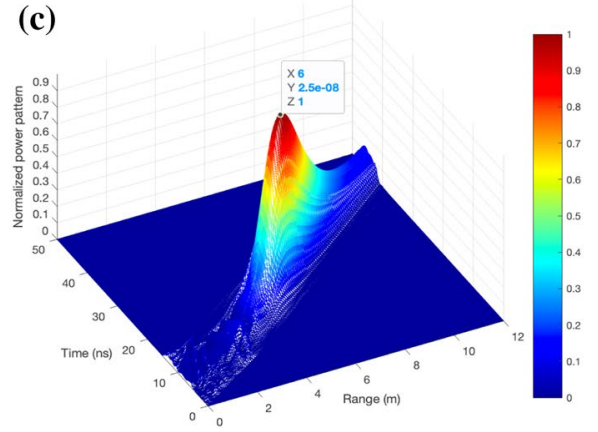

Fig. 12 Significance of the wave propagation effect. a C-TMOFO FDA original time-invariant beampattern. $\mathbf{b}$ C-TMOFO FDA corrected time-variant beampattern. c Original time-variant beampattern of the short-range FDA radar based on arc-tangent function

\subsection{Applications}

While most current research activities mainly focus on radar applications, such as localization, FDA is also very appealing for communication applications. The security issue in wireless communication systems is of great concern due to its broadcasting nature of transmission [29]. In this scenario, the communications between the transmitter and the legitimate destination receiver are more likely to be intercepted easily by eavesdroppers. Therefore, an active research topic has been focused on how to prevent eavesdroppers access to the desired information in wireless communication systems.

Directional modulation (DM) is recognized as one of the most promising techniques in the point-to-point physical layer secure communications [147]. DM projects digitally modulated signals into a pre-defined secure communication spatial direction, while simultaneously distorting the constellation of these signals in all other directions [148]. This can significantly decrease the risk of these signals being eavesdropped. As such, DM techniques using PA antenna have been widely applied in wireless communications, which only achieves one-dimension security, i.e., angle-dependent directional modulation [149]. Therefore, the PA based DM techniques are less successful when an eavesdropper and the legitimate user are located in the same direction.

On the other hand, frequency diversity has been shown to be a highly effective technique in wireless communications, which provides a number of advantages, including increased system capacity and SNR, security and robustness. Motivated by the rangeangle dependent beampattern of FDAs, researches have been carried out on FDA based 
DM techniques to enhance physical layer security of wireless communications [150, 151]. Both the PA and FDA based DM techniques can be typically applied to secure communication in the line-of-propagation channels such as millimeter wave, satellite communications, unmanned aerial vehicles, and smart transportation. Compared with the PA-based DM techniques, the FDA based DM technique can achieve the physical layer security in both range and angle dimensions. Since the FDA apparent scan angle is different than its nominal scan angle, precise beam steering depends on both the range and the angle. Consequently, the actual beam steering direction in FDAs cannot be effectively predicted as conventional phased arrays. Equation (22) demonstrates how the scan angle changed with frequency increment and generated an apparent scan angle.The FDA based DM techniques have provable performance guarantee when the legitimate user and eavesdropper locate in the same direction but different ranges [152].

Until recently, several attractive FDA based DM techniques have been proposed to ensure secure communication with low probability of interception and low probability of detection. To name a few, a decoupled range-angle dependent DM scheme using FDA with symmetrically and nonlinearly increasing frequency offsets is proposed in [150] for secure wireless communications. In [151], frequency offsets optimized with genetic algorithm are used to achieve the physical layer security in both angle and range dimensions. Two time-invariant range-angle dependent DM schemes based on time-modulated frequency offsets FDA were proposed in [152] to achieve time-invariant spatial fine focusing point-to-point physical layer secure communications. In the DM transmission schemes, artificial noise plays a pivotal role. In artificial noise-aided secure transmission, the transmitter also sends artificial noise as an interference signal of eavesdropper, which only interferes the eavesdropper without affecting the legitimate user. The DM with artificial noise based on random FDA, referred to as the RFDA-DM-AN scheme, is presented in [153] to enhance physical layer security of wireless communications. Similarly, DM with an artificial-noise-aided approach, and FDA beamforming-based approach are, respectively, proposed for the security of proximal legitimate user and eavesdropper in [154] and [155]. In contrast to the single-user FDA-DM schemes, multi-beam DM synthesis based on FDA is investigated in [156-159].

As such, DM utilizing FDA is shown to be more robust than using PA antenna, and becomes a preferred choice to achieve enhanced physical layer secure communications. Therefore, FDA based DM has been an intensive research topic, owing to their superior performance to the PA based DM techniques [160-164]. Similar to FDA, a new transmit diversity technique, namely, hybrid code, is presented in [165], where the operation of the PA radar and the functionality of the coherent MIMO radar are unified for use in electronically steered phased array radars. The hybrid code demonstrates low range sidelobes and better angular selectivity. Instead of scanning, the hybrid code obtains the transmit beampatterns for any/all chosen angular directions by signal processing means. The hybrid code is a promising concept that can play an important role in future radar, and communication applications.

\section{Future work}

This work has looked at broad concepts that provide a basis for continuing research in FDAs. In particular, areas that are suitable for continuing research includes: 
1 Optimization of array geometry: Further research may explore optimal geometry for FDA radars. Various array configurations merit further consideration, including parabolic geometries and multi-ring UCA.

2 Optimization of frequency offsets: More investigations should be carried out to optimally design computationally efficient FDA frequency offsets.

3 Waveform optimization: Most of the investigations to date have focused on FDAs with narrowband signal sources, further efforts are required to its application in wideband and ultra-wideband systems.

4. Optimal signal processing: FDAs should be researched in more complex electromagnetic environment such as mainlobe and sidelobe clutters, jamming, and intense interference environment.

5 Advanced applications: Much of the recent work in FDAs has been focused on localization related applications such as navigation and radar. Although some studies discuss their application in various other fields including communication, more research is required to explore its advantages in other fields.

6 Wave Propagation: To mitigate the performance degradation caused by wave propagation, and ensure the applicability of FDA radars in practical applications, improved time-variant focusing techniques with longer dwell time is an emerging research direction in FDAs.

7 Multi-target localization: Although various FDAs with time-variant consideration is proposed for a single target scenario, extension to multi-target detection may also be included in future studies.

8 Hardware Implementation: A future effort may also focus on implementation of time-variant FDAs with hardware implementation.

\section{Conclusion}

This paper provides an overview of the FDA radar, and briefly discuss its performance and implementation issues with an emphasis on recent research. The FDA radar is a newly emerging concept, which has a range-angle dependent beampattern. Unlike PA radars, which work with fixed carrier frequencies, the FDA radar employ a small frequency increment across the array elements to achieve DOFs in both the angle and range dimensions. We have briefly analyzed the characteristics of the FDA beampattern from its origin to the current state-of-the-art. Many different approaches to FDA radar for improved performance have been discussed briefly. The conventional FDA radar using progressive incremental frequency offsets yields a range-angle coupled beampattern. To decouple the FDA beampattern in range-angle dimensions, nonlinear arrays or nonlinear frequency offsets were utilized. However, the resulting beampatterns exhibit time-periodicity. To deal with these time-periodic beampatterns, time-modulated frequency offsets were reported, which achieve range-angle decoupled, and time-invariant beampatterns at the target position. However, some recent studies indicate that the propagation process of transmitted signals was ignored in the FDA literature. The FDA beampattern is always time-variant in free space. Therefore, the focused beampattern for FDAs using time-invariant techniques cannot be realized practically. Although some researchers were successful in focusing the transmit energy over the desired target 
location, the dwell-time is very short. Future work will likely address the time-variant focusing problem with longer dwell time, and extension to multi-target scenarios is also a subject for future work. FDAs were also shown to have provable performance in wireless communication applications such as physical layer secure point-to-point communication. However, unlike the PA which is a mature technology, FDA has yet to achieve super resolution capabilities, and many practical problems still need to be investigated.

\begin{abstract}
Abbreviations
AF: Array factor; DOFs: Degrees-of-freedom; DM: Directional modulation; EM: Electromagnetic; ESA: Electronically steerable arrays; FDA: Frequency diverse array; HPBW: Half-power beamwidth; MIMO: Multi-input multi-output; OFDM: Orthogonal frequency division multiplexing; OSNFO: Optimized static nonlinear frequency offset; PA: Phased array; P-SLL: Peak sidelobe level; SFP-FDA: Stepped frequency pulse FDA; STAP: Space-time adaptive processing; TDFO: Time-dependent frequency offsets; TMDP-FDA: Time-modulated double parameter FDA; TMLFO: Time-modulated logarithmically increasing frequency offset; TMOFO-FDA:: Time-modulated optimized frequency offset FDA; UC-FDA: Uniform circular frequency diverse array; ULA: Uniform linear array; 3D: Three-dimensional.
\end{abstract}

\title{
Acknowledgements
}

Not applicable.

\section{Authors' contributions}

ZA: Conceptualization, Formal analysis, Investigation, Methodology, Validation, Writing-original draft. MC: Validation, Revision, Proof-reading \& editing. SB: Funding acquisition, Supervision, Validation, Writing-review \& editing. All authors read and approved the final manuscript.

\section{Funding}

This work was supported in part by the National Natural Science Foundation of China under Grant 61671260.

Availability of data and materials

Not applicable.

\section{Declarations}

Competing interests

The authors declare that they have no competing interests.

Received: 5 April 2021 Accepted: 21 October 2021

Published online: 16 November 2021

\section{References}

1. S. Haykin, Array Processing: Applications to Radar (Dowden) (Hutchinson \& Ross, Stroudsburg, 1980)

2. S. Haykin, J. Litva, T.J. Shepherd, Radar Array Processing (Springer, New York, 1993)

3. A.L. Swindlehurst, P. Stoica, Maximum likelihood methods in radar array signal processing. Proc. IEEE 86(2), 421-441 (1998). https://doi.org/10.1109/5.659495

4. R.J. Vaccaro, The past, present, and the future of underwater acoustic signal processing. IEEE Signal Process. Mag. 15(4), 21-51 (1998). https://doi.org/10.1109/79.689583

5. E.Y. Gorodetskaya, A.I. Malekhanov, A.G. Sazontov, N.K. Vdovicheva, Deep-water acoustic coherence at long ranges: theoretical prediction and effects on large-array signal processing. IEEE J. Ocean. Eng. 24(2), 156-171 (1999). https://doi.org/10.1109/48.757268

6. Q.-G. Liu, B. Champagne, P. Kabal, A microphone array processing technique for speech enhancement in a reverberant space. Speech Commun. 18(4), 317-334 (1996). https://doi.org/10.1016/0167-6393(96)00011-8

7. L.C. Godara, Application of antenna arrays to mobile communications. II. Beam-forming and direction-of-arrival considerations. Proc. IEEE 85(8), 1195-1245 (1997). https://doi.org/10.1109/5.622504

8. J.E. Evans, J.R. Johnson, D.F. Sun, Application of Advanced Signal Processing Techniques to Angle of Arrival Estimation in ATC Navigation and Surveillance Systems (M.I.T. Lincoln Laboratory, Technical Report (1982)

9. A. Nehorai, B. Porat, E. Paldi, Detection and localization of vapor-emitting sources. IEEE Trans. Signal Process. 43(1), 243-253 (1995). https://doi.org/10.1109/78.365304

10. A. Gershman, V. Turchin, Nonwave field processing using sensor array approach. Signal Process. 44(2), 197-210 (1995). https://doi.org/10.1016/0165-1684(95)00024-8

11. A. Jeremic, A. Nehorai, Landmine detection and localization using chemical sensor array processing. IEEE Trans. Signal Process. 48(5), 1295-1305 (2000). https://doi.org/10.1109/78.839977

12. R.J. Mailloux, Electronically scanned arrays. Synth. Lect. Anten. 2(1), 1-82 (2007). https://doi.org/10.2200/S0008 1ED1V01Y200612ANT006

13. M.A. Richards, Fundamentals of Radar Signal Processing (McGraw-Hill, New York, 2005), pp. 1-22

14. M.I. Skolnik, Introduction to Radar Systems, 3rd edn. (McGraw-Hill, New York, 2001) 
15. R.C. Hansen, Microwave Scanning Antenna, Volume I Apertures (Academic, New York, 1964)

16. R.C. Hansen, Microwave scanning antenna, Volume II Array Theory and Practice (Academic, New York, 1966)

17. R.C. Hansen, Microwave scanning antenna, Volume III Array Systems (Academic, New York, 1966)

18. T.K. Sarkar, R.J. Mailloux, A.A. Oliner, M. Salazar-Palma, D.L. Sengupta, History of Wireless (Wiley, New York, 2006)

19. A.J. Fenn, D.H. Temme, W.P. Delaney, W.E. Courtney, The development of phased array radar technology. Linc. Lab. J. 12(2), 321-340 (2000)

20. R.C. Hansen, Phased Array Antennas, 2nd edn. (Wiley, New York, 2009), pp. 7-47

21. R.C. Hansen, Significant Phased Array Papers (Artech House, Norwood, 1973)

22. E. Brookner, Practical Phased-Array Antenna Systems (Artech House, Norwood, 1991)

23. E. Brookner, Phased array radars-past, present and future. in Proceedings of RADAR 2002, Edinburgh, UK (2002), pp. 104-113. https://doi.org/10.1109/RADAR.2002.1174663

24. R.J. Mailloux, Phased Array Antenna Handbook (Artech House, Norwood, 1994)

25. J. Li, P. Stoica, The phased array is the maximum SNR active array. IEEE Signal Process. Mag. 27(2), 143-144 (2010). https://doi.org/10.1109/MSP.2009.935418

26. D. Parker, D.C. Zimmermann, Phased arrays-Part II: implementations, applications, and future trends. IEEE Trans. Microw. Theory Technol. 50(3), 688-698 (2002). https://doi.org/10.1109/22.989954

27. A. Basit, W. Khan, S. Khan, I.M. Qureshi, Development of frequency diverse array radar technology: a review. IET Radar Sonar Navig. 12(2), 165-175 (2018). https://doi.org/10.1049/iet-rsn.2017.0207

28. A.J. Fenn, Adaptive Antenna and Phased Arrays for Radar and Communications (Artech House, Norwood, 2008)

29. S.Y. Nusenu, A. Basit, Frequency diverse array antennas: From their origin to their application in wireless communication systems. J. Comput. Netw. Commun. 2018, 5815678 (2018). https://doi.org/10.1155/2018/5815678

30. H.E. Shanks, A new technique for electronic scanning. IRE Trans. Antennas Propag. 9(2), 162-166 (1961). https://doi.org/10.1109/TAP.1961.1144965

31. G. Oliveri, M. Carlin, The new role of time-modulation for innovative array synthesis. in Proceedings of Fifth European Conference on Antennas and Propagation (EUCAP), Rome, Italy (2011), pp. 2209-2212

32. S.K. Mandal, G.K. Mahanti, G. Rowdra, Synthesis of flat top beam pattern through time modulation of linear array. in Proceedings of the IEEE International Conference on Signal Processing, Communication and Computing, Hong Kong (2012), pp. 32-37. https://doi.org/10.1109/ICSPCC.2012.6335645

33. K. Koh, H. Elyasi, Time-interleaved phased arrays with parallel signal processing in RF modulation. IEEE Trans. Antennas Propag. 62(2), 677-689 (2014). https://doi.org/10.1109/TAP.2013.2293332

34. A. Ishimaru, H.-S. Tuan, Theory of frequency scanning of antennas. IRE Trans. Antennas Propag. 10(2), 144-150 (1962). https://doi.org/10.1109/TAP.1962.1137834

35. K.L. Klohn, R.E. Horn, H. Jacobs, E. Freibergs, Silicon waveguide frequency scanning linear array antenna. IEEE Trans. Microw. Theory Technol. 26(10), 764-773 (1978). https://doi.org/10.1109/TMTT.1978.1129484

36. M.H. Mazaheri, M. Fakharzadeh, M. Akbari, S. Safavi-Naeini, A figure of merit in a time-modulated array. IEEE Antennas Wirel. Propag. Lett. 18(10), 2086-2089 (2019). https://doi.org/10.1109/LAWP.2019.2937755

37. R. Gahley, B. Basu, A time modulated printed dipole antenna array for beam steering application. Int. J. Antennas Propag. 2017, 3687293 (2017). https://doi.org/10.1155/2017/3687293

38. M.C. Wicks, P. Antonik, Frequency diverse array with independent modulation of frequency, amplitude, and phase. U.S. Patent 7,319,427 (2008)

39. A. Aytun, Frequency diverse array radar. Master's thesis, Naval Postgraduate School (2010)

40. S. Brady, Frequency diverse array radar: signal characterization and measurement accuracy. Master's thesis, Air Force Institute of Technology (2010)

41. P. Antonik, M.C. Wicks, H.D. Griffiths, C.J. Baker, Frequency diverse array radars. in Proceedings of IEEE Radar Conference, Verona, NY, USA (2006), pp. 215-217. https://doi.org/10.1109/RADAR.2006.1631800

42. J. Huang, K.-F. Tong, C.J. Baker, Frequency diverse array with beam scanning feature. in Proceedings of IEEE Antennas Propagation Conference, San Diego, California (2008), pp. 1-4. https://doi.org/10.1109/APS.2008. 4619415

43. P. Antonik, An investigation of a frequency diverse array. Ph.D. dissertation, University College London (2009)

44. W.-Q. Wang, Overview of frequency diverse array in radar and navigation applications. IET Radar Sonar Navig. 10(6), 1001-1012 (2016). https://doi.org/10.1049/iet-rsn.2015.0464

45. W.-Q. Wang, Frequency diverse array antenna: new opportunities. IEEE Antennas Propag. Mag. 57(2), 145-152 (2015). https://doi.org/10.1109/MAP.2015.2414692

46. H. Zeng, Z. Ahmad, J. Zhou, Q. Wang, Y. Wang, DOA estimation algorithm based on adaptive filtering in spatial domain. China Commun. 13(12), 49-58 (2016). https://doi.org/10.1109/CC.2016.7897554

47. R.F. Tigrek, W.J.A. De Heij, P. Van Genderen, OFDM signals as the radar waveform to solve Doppler ambiguity. IEEE Trans. Aerosp. Electron. Syst. 48(1), 130-143 (2012). https://doi.org/10.1109/TAES.2012.6129625

48. Z. Wang, F. Tigrek, O. Krasnov, F. Van Der Zwan, P. Van Genderen, A. Yarovoy, Interleaved OFDM radar signals for simultaneous polarimetric measurements. IEEE Trans. Aerosp. Electron. Syst. 48(3), 2085-2099 (2012). https:// doi.org/10.1109/TAES.2012.6237580

49. T. Zhang, X. Xia, L. Kong, IRCI free range reconstruction for SAR imaging with arbitrary length OFDM pulse. IEEE Trans. Signal Process. 62(18), 4748-4759 (2014). https://doi.org/10.1109/TSP.2014.2339796

50. G. Cui, H. Li, M. Rangaswamy, MIMO radar waveform design with constant modulus and similarity constraints. IEEE Trans. Signal Process. 62(2), 343-353 (2014). https://doi.org/10.1109/TSP.2013.2288086

51. W. Huleihel, J. Tabrikian, R. Shavit, Optimal adaptive waveform design for cognitive MIMO radar. IEEE Trans. Signal Process. 61(20), 5075-5089 (2013). https://doi.org/10.1109/TSP.2013.2269045

52. S. Ahmed, M.-S. Alouini, MIMO-radar waveform covariance matrix for high SINR and low side-lobe levels. IEEE Trans. Signal Process. 62(8), 2056-2065 (2014). https://doi.org/10.1109/TSP.2014.2307282

53. F.S. Johansson, L.G. Josefsson, T. Lorentzon, A novel frequency scanned reflector antenna. IEEE Trans. Signal Process. 37(8), 984-989 (1989). https://doi.org/10.1109/8.34134 
54. C. Vázquez, C. García, Y. Álvarez, S. Ver-Hoeye, F. Las-Heras, Near field characteristics of an imaging system based on a frequency scanning antenna array. IEEE Trans. Signal Process. 61(5), 2874-2879 (2013). https://doi.org/10.1109/ TAP.2013.2244834

55. J. Fondevila, J.C. Bregains, F. Ares, E. Moreno, Optimizing uniformly excited linear arrays through time modulation. IEEE Antennas Wirel. Propag. Lett. 3, 298-301 (2004). https://doi.org/10.1 109/LAWP.2004.838833

56. P. Rocca, L. Manica, L. Poli, A. Massa, Synthesis of compromise sum-difference arrays through time-modulation. IET Radar Sonar Navig. 3(6), 630-637 (2009). https://doi.org/10.1049/iet-rsn.2009.0058

57. J. Dorey, Y. Blanchard, F. Christophe, The RIAS project, a new approach to air surveillance radar. in Proceedings of SEE Colloque International sur le Radar, Versailles, France (1984) ONERA, TP, no. 1984-20, p. 7. In French

58. J. Dorey, G. Garnier, G. Auvray, RIAS, synthetic impulse and antenna radar. in Proceedings of International Conference Radar, Paris, France (1989), pp. 556-562

59. A. Luce, H. Molina, D. Muller and V. Thirard, Experimental results on RIAS digital beamforming radar. in Proceedings of International Conference on Radar, Brighton, UK (1992), pp. 74-77

60. C. Baixiao, Z. Shouhong, W. Yajun and W. Jun, Analysis and experimental results on sparse-array synthetic impulse and aperture radar. in Proceedings of CIE International Conference Radar, Beijing, China (2001), pp. 76-80. https:// doi.org/10.1109/ICR.2001.984627

61. G. Zhao, B. Chen, S. Zhu, Direction synthesis in DOA estimation for monostatic multiple input multiple output (MIMO) radar based on synthetic impulse and aperture radar (SIAR) and its performance analysis. Sci. China Ser. E-Technol. Sci. 51, 656 (2008). https://doi.org/10.1007/s11431-008-0048-2

62. K.W. Forsythe, D.W. Bliss, in MIMO Radar Signal Processing, edited by J. Li and P. Stoica (Wiley, 2008)

63. R. Li, J. Li, W. Zhang, Z. He, Reduced-dimension space-time adaptive processing based on angle-Doppler correlation coefficient. J. Adv. Signal Process. 2016, 97 (2016). https://doi.org/10.1186/s13634-016-0395-2

64. I.J. Gupta, T.D. Moore, Space-frequency adaptive Processing (SFAP) for radio frequency interference mitigation in spread-spectrum receivers. IEEE Trans. Antennas Propag. 52(6), 1611-1615 (2004). https://doi.org/10.1109/TAP. 2004.829850

65. Z. Ahmad, Y. Song, Q. Du, Wideband DOA estimation based on incoherent signal subspace method. Compel-Int. J. Comput. Math. Electr. Electron. Eng. 37(3), 1271-1289 (2018). https://doi.org/10.1108/COMPEL-10-2017-0443

66. Z. Ahmad, Y. Song, Q. Du, Adaptive wideband beamforming based on digital delay filter. J. Microw. Optoelectron. Electromagn. Appl. 15(3), 262-274 (2016). https://doi.org/10.1590/2179-10742016v15i3630

67. P. Antonik, M.C. Wicks, H.D. Griffiths, C.J. Baker, Range dependent beamforming using element level waveform diversity. in Proceedings of the International Waveform Diversity Design Conference, Lihue, HI, USA (2006), pp. 1-6. https://doi.org/10.1109/WDD.2006.8321488

68. M. Secmen, S. Demir, A. Hizal, T. Eker, Frequency diverse array antenna with periodic time modulated pattern in range and angle. in Proceedings of the IEEE Radar Conference, Waltham, MA, USA (2007), pp. 427-430, https://doi. org/10.1109/RADAR.2007.374254

69. J. Huang, K. Tong, C. Baker, Frequency diverse array: Simulation and design. in Proceedings of the LAPS Antennas and Propagation Conference, Loughborough, UK (2009), pp. 253-256. https://doi.org/10.1109/LAPC.2009.5352422

70. L. Zhuang, X. Liu, W. Yu, Precisely beam steering for frequency diverse arrays based on frequency offset selection. in Proceedings of the International Radar Conference, Bordeaux, France (2009), pp. 1-4

71. W.-Q. Wang, H. Shao, J. Cai, Range-angle-dependent beamforming by frequency diverse array antenna. Int. J. Antennas Propag. 2012, 760489 (2012). https://doi.org/10.1155/2012/760489

72. M.C. Wicks, P. Antonik, Method and apparatus for a frequency diverse array. U.S. Patent 7.511,665B2 (2009)

73. T. Higgins, S.D. Blunt, Analysis of range-angle coupled beamforming with frequency-diverse chirps. in Proceedings of 4th International Waveform Diversity Design Conference, Orlando, FL, USA (2009), pp. 140-144. https://doi.org/10. 1109/WDDC.2009.4800333

74. T. Eker, S. Demir, A. Hizal, Exploitation of linear frequency modulated continuous waveform (LFMCW) for frequency diverse arrays. IEEE Trans. Antennas Propag. 61(7), 3546-3554 (2013). https://doi.org/10.1109/TAP.2013.2258393

75. P. Baizert, T.B. Hale, M.A. Temple, M.C. Wicks, Forward- looking radar GMTI benefits using a linear frequency diverse array. Electron. Lett. 42(22), 1311-1312 (2006). https://doi.org/10.1049/el:20062791

76. P.F. Sammartino, C.J. Baker, H.D. Griffiths, Frequency diverse MIMO techniques for radar. IEEE Trans. Aerosp. Electron. Syst. 49(1), 201-222 (2013). https://doi.org/10.1109/TAES.2013.6404099

77. P.F. Sammartino, C.J. Baker, Developments in the frequency diverse bistatic system. in Proceedings of IEEE Radar Conference, Pasadena, CA (2009), pp. 1-5. https://doi.org/10.1109/RADAR.2009.4976937

78. J. Farooq, M.A. Temple, M.A. Saville, Application of frequency diverse arrays to synthetic aperture radar imaging. in Proceedings of International Electromagnetic Advance Application Conference, Turin, Italy (2007), pp. 447-449. https:// doi.org/10.1109/ICEAA.2007.4387334

79. J. Farooq, M.A. Temple, M.A. Saville, Exploiting frequency diverse array processing to improve SAR image resolution. in Proceedings of IEEE Radar Conference, Rome, Italy (2008), pp. 1-5. https://doi.org/10.1 109/RADAR.2008. 4721083

80. J. Farooq, Frequency diversity for improving synthetic aperture radar imaging. Ph.D. dissertation, Department of Air Force Air University, Institute of Technology, Wright-Patterson AFB, OH, USA (2009)

81. W.-Q. Wang, Phased-MIMO radar with frequency diversity for range-dependent beamforming. IEEE Sensors $J$. 13(4), 1320-1328 (2013). https://doi.org/10.1109/JSEN.2012.2232909

82. C. Cetintepe, S. Demir, Multipath characteristics of frequency diverse arrays over a ground plane. IEEE Trans. Antennas Propag. 62(7), 3567-3574 (2014). https://doi.org/10.1109/TAP.2014.2316292

83. Y. Wang, W.-Q. Wang, H. Shao, Frequency diverse array radar Cramer-Rao lower bounds for estimating direction, range, and velocity. Int. J. Antennas Propag. 2014, 830869 (2014). https://doi.org/10.1155/2014/830869

84. W.-Q. Wang, H.C. So, Transmit subaperturing for range and angle estimation in frequency diverse array radar. IEEE Trans. Signal Process. 62(8), 2000-2011 (2014). https://doi.org/10.1109/TSP.2014.2305638

85. W.-Q. Wang, Two-dimensional imaging of targets by stationary frequency diverse array. Remote Sens. Lett. 4(11), 1067-1076 (2013). https://doi.org/10.1080/2150704X.2013.837228 
86. J. Xu, S. Zhu, G. Liao, Space-time-range adaptive processing for airborne radar systems. IEEE Sens. J. 15(3), 1602-1610 (2015). https://doi.org/10.1109/JSEN.2014.2364594

87. J. Xu, G. Liao, S. Zhu, L. Huang, H.C. So, Joint range and angle estimation using MIMO radar with frequency diverse array. IEEE Trans. Signal Process. 63(13), 3396-3410 (2015). https://doi.org/10.1109/TSP.2015.2422680

88. W.-Q. Wang, H. Shao, Range-angle localization of targets by a double-pulse frequency diverse array radar. IEEE J. Sel. Top. Signal Process. 8(1), 106-114 (2014). https://doi.org/10.1109/JSTSP.2013.2285528

89. M. Tan, C.Y. Wang, Z.-H. Li, X. Li, L. Bao, Stepped frequency pulse frequency diverse array radar for target localization in angle and range domains. Int. J. Antennas Propag. 2018, 8962048 (2018). https://doi.org/10.1155/2018/89620 48

90. W. Khan, I.M. Qureshi, A. Basit, M. Zubair, A double pulse MIMO frequency diverse array radar for improved range-angle localization of target. Wirel. Pers. Commun. 82(4), 2199-2213 (2015). https://doi.org/10.1007/ S11277-015-2342-1

91. J. Xu, G. Liao, H.C. So, Space-time adaptive processing with vertical frequency diverse array for range-ambiguous clutter suppression. IEEE Trans. Geosci. Remote Sens. 54(9), 5352-5364 (2016). https://doi.org/10.1109/TGRS.2016. 2561308

92. K. Gao, H. Shao, J. Cai, H. Chen, W.-Q. Wang, Frequency diverse array MIMO radar adaptive beamforming with range-dependent interference suppression in target localization. Int. J. Antennas Propag. 2015, 358582 (2015). https://doi.org/10.1155/2015/358582

93. K. Gao, W.-Q. Wang, J. Cai, Frequency diverse array and MIMO hybrid radar transmitter design via Cramer-Rao lower bound minimisation. IET Radar Sonar Navig. 10(9), 1660-1670 (2016). https://doi.org/10.1049/iet-rsn.2015.0644

94. W.-Q. Wang, H.C. So, H. Shao, Nonuniform frequency diverse array for range-angle imaging of targets. IEEE Sens. J. 14(8), 2469-2476 (2014). https://doi.org/10.1109/JSEN.2014.2304720

95. Y. Liao, W.-Q. Wang, Z. Zheng, Frequency diverse array beampattern synthesis using symmetrical logarithmic frequency offsets for target indication. IEEE Trans. Antennas Propag. 67(5), 3505-3509 (2019). https://doi.org/10. 1109/TAP.2019.2900353

96. M. Tan, C. Wang, Z. Li, J. Bai, L. Bao, Adaptive beamforming using frequency diverse MIMO radar with nonlinear frequency offset. in IEEE 11th Sensor Array and Multichannel Signal Processing Workshop (SAM), Hangzhou, China (2020), pp. 1-5

97. L. Lan, G. Liao, J. Xu, J. Wen, Range-angle pencil-beamforming for non-uniformly distributed array radar. Multidimens. Syst. Signal Process. 29, 867-886 (2018). https://doi.org/10.1007/s11045-017-0477-9

98. K. Gao, W. Wang, J. Cai, J. Xiong, Decoupled frequency diverse array range angle dependent beampattern synthesis using non-linearly increasing frequency offsets. IET Microw. Antennas Propag. 10(8), 880-884 (2016). https:// doi.org/10.1049/iet-map.2015.0658

99. A. Basit, I.M. Qureshi, W. Khan, S. Rehman, M.M. Khan, Beam pattern synthesis for an FDA radar with Hamming window-based nonuniform frequency offset. IEEE Antennas Wirel. Propag. Lett. 16, 2283-2286 (2017). https://doi. org/10.1109/LAWP.2017.2714761

100. Z. Wang, W.-Q. Wang, H. Shao, Range-azimuth decouple beamforming for frequency diverse array with Costassequence modulated frequency offsets. EURASIP J. Adv. Signal Process. 2016(1), 124 (2016). https://doi.org/10. 1186/s13634-016-0422-3

101. M. Mahmood, H. Mir, FDA transmit beampattern synthesis using piecewise trigonometric frequency offset. IET Radar Sonar Navig. 13(7), 1149-1153 (2019). https://doi.org/10.1049/iet-rsn.2018.5501

102. M. Mahmood, H. Mir, Frequency diverse array beamforming using nonuniform logarithmic frequency increments. IEEE Antennas Wirel. Propag. Lett. 17(10), 1817-1821 (2018). https://doi.org/10.1109/LAWP.2018.2867085

103. Z. Wang, T. Mu, Y. Song et al., Beamforming of frequency diverse array radar with nonlinear frequency offset based on logistic map. Prog. Electromagn. Res. M. 64, 55-63 (2018)

104. H. Shao, J. Dai, J. Xiong, H. Chen, W.-Q. Wang, Dot-shaped range-angle beampattern synthesis for frequency diverse array. IEEE Antennas Wirel. Propag. Lett. 15, 1703-1706 (2016). https://doi.org/10.1109/LAWP.2016.2527818

105. W. Khan, I.M. Qureshi, A. Basit, W. Khan, Range-Bins-Based MIMO frequency diverse array radar with logarithmic frequency offset. IEEE Antennas Wirel. Propag. Lett. 15, 885-888 (2016). https://doi.org/10.1109/LAWP.2015.24789 64

106. W. Khan, I.M. Qureshi, S. Saeed, Frequency diverse array radar with logarithmically increasing frequency offset. IEEE Antennas Wirel. Propag. Lett. 14, 499-502 (2015). https://doi.org/10.1109/LAWP.2014.2368977

107. A. Basit, I.M. Qureshi, W. Khan et al., Cognitive frequency diverse array radar with symmetric non-uniform frequency offset. Sci. China Inf. Sci. 59, 102314 (2016). https://doi.org/10.1007/s11432-015-0503-x

108. H. Shao, J. Li, H. Chen, W.-Q. Wang, Adaptive frequency offset selection in frequency diverse array radar. IEEE Antennas Wirel. Propag. Lett. 13, 1405-1408 (2014). https://doi.org/10.1109/LAWP.2014.2340893

109. Y. Liao, H. Tang, X. Chen, W.-Q. Wang, Frequency diverse array beampattern synthesis with Taylor windowed frequency offsets. IEEE Antennas Wirel. Propag. Lett. 19(11), 1901-1905 (2020). https://doi.org/10.1109/LAWP.2020. 3024710

110. J. Li, S. Ouyang, K. Liao, X. Sun, Identifying unambiguous frequency patterns for two-target localization using frequency diverse array. Signal Process. 171, 107452 (2020). https://doi.org/10.1016/j.sigpro.2019.107452

111. S.L. Wang, Z. Xu, X. Liu, W. Dong, G. Wang, Subarray-based frequency diverse array for target range-angle localization with monopulse processing. IEEE Sens. J. 18(14), 5937-5947 (2018). https://doi.org/10.1109/JSEN.2018.28442 80

112. W.-G. Tang, H. Jiang, Q. Zhang, Range-angle decoupling and estimation for FDA-MIMO radar via atomic norm minimization and accelerated proximal gradient. IEEE Signal Process. Lett. 27, 366-370 (2020). https://doi.org/10. 1109/LSP.2020.2972470

113. J. Xiong, W. Wang, H. Shao, H. Chen, Frequency diverse array transmit beampattern optimization with genetic algorithm. IEEE Antennas Wirel. Propag. Lett. 16, 469-472 (2017). https://doi.org/10.1109/LAWP.2016.2584078

114. W. Khan, I.M. Qureshi, Frequency diverse array radar with time-dependent frequency offset. IEEE Antennas Wirel. Propag. Lett. 13, 758-761 (2014). https://doi.org/10.1109/LAWP.2014.2315215 
115. Y. Xu, X. Shi, J. Xu, P. Li, Range-angle-dependent beamforming of pulsed frequency diverse array. IEEE Trans. Antennas Propag. 63(7), 3262-3267 (2015). https://doi.org/10.1109/TAP.2015.2423698

116. A.-M. Yao, W. Wu, D.G. Fang, Frequency diverse array antenna using time-modulated optimized frequency offset to obtain time-invariant spatial fine focusing beampattern. IEEE Trans. Antennas Propag. 64(10), 4434-4446 (2016). https://doi.org/10.1109/TAP.2016.2594075

117. A.-M. Yao, W. Wu, D.G. Fang, Solutions of time-invariant spatial focusing for multi-targets using time modulated frequency diverse antenna arrays. IEEE Antennas Wirel. Propag. Lett. 65(2), 552-566 (2017). https://doi.org/10. 1109/TAP.2016.2633902

118. Q. Cheng, J. Zhu, T. Xie, J. Luo, Z. Xu, Time-invariant angle-range dependent directional modulation based on timemodulated frequency diverse arrays. IEEE Access 5, 26279-26290 (2017). https://doi.org/10.1109/ACCESS.2017. 2772246

119. A.-M. Yao, P. Rocca, W. Wu, A. Massa, D.G. Fang, Synthesis of time-modulated frequency diverse arrays for shortrange multi-focusing. IEEE J. Sel. Top. Signal Process. 11(2), 282-294 (2017). https://doi.org/10.1109/JSTSP.2016. 2615267

120. T. Mu, Y. Song, Z. Wang, Beampattern synthesis for frequency diverse array based on time-modulated double parameters approach. J. Microw. Optoelectron. Electromagn. Appl. 17(3), 352-365 (2018). https://doi.org/10.1590/ 2179-10742018v17i31240

121. H. Shao, X. Li, W. Wang, J. Xiong, H. Chen, Time-invariant transmit beampattern synthesis via weight design for FDA radar. in Proceedings of IEEE Radar Conference, Philadelphia, PA, USA (2016), pp. 1-4. https://doi.org/10.1 109/RADAR 2016.7485212

122. Y. Gu, Y.-D. Zhang, Compressive sampling optimization for user signal parameter estimation in massive MIMO systems. Digit. Signal Process. 94, 105-113 (2019). https://doi.org/10.1016/j.dsp.2019.06.010

123. S. Qin, Y.-D. Zhang, M.G. Amin, F. Gini, Frequency diverse coprime arrays with coprime frequency offsets for multitarget localization. IEEE J. Sel. Top. Signal Process. 11(2), 321-335 (2017). https://doi.org/10.1109/JSTSP.2016.26271 84

124. Y. Liu, H. Ruan, L. Wang, A. Nehorai, The random frequency diverse array: a new antenna structure for uncoupled direction-range indication in active sensing. IEEE J. Sel. Topics Signal Process. 11(2), 295-308 (2017). https://doi. org/10.1109/JSTSP.2016.2627183

125. C. Wang, X. Zhang, J. Li, FDA-MIMO radar for 3D localization: virtual coprime planar array with unfolded coprime frequency offset framework and TRD-MUSIC algorithm. Digit. Signal Process. 120, 103017 (2021). https://doi.org/ 10.1016/j.dsp.2021.103017

126. W. Chu, Y. Liu, X. Li, Y. Zhao, Q. Zhang, F. Yan, Improving target detection ability based on time invariant and dot-shape beamforming in TMRC-FDA-MIMO radar. Wireless Pers. Commun. (2021). https://doi.org/10.1007/ s11277-021-08240-6

127. S. Saeed, I.M. Qureshi, W. Khan, A. Salman, An investigation into uniform circular frequency diverse array (UCFDA) radars. Remote Sens. Lett. 6(9), 707-714 (2015). https://doi.org/10.1080/2150704X.2015.1069903

128. S. Saeed, I.M. Qureshi, W. Khan, A. Salman, Elliptical frequency diverse array radars: uniform and non-uniform frequency offsets. Remote Sens. Lett. 8(5), 478-487 (2017). https://doi.org/10.1080/2150704X.2017.1280623

129. Y. Xu, X. Shi, J. Xu, W. Li, Beampattern analysis of planar frequency diverse array. Int. J. RF Microwave Comput. Aided Eng. 25(5), 436-444 (2015). https://doi.org/10.1002/mmce.20881

130. Flat-top beampattern synthesis in range and angle domains for frequency diverse array via second-order cone programming. IEEE Antennas Wirel. Propag. Lett. 15, 1479-1482 (2016). https://doi.org/10.1109/LAWP.2015.25137 58

131. Y. Xu, X. Shi, J. Xu, L. Huang, W. Li, Range-angle-decoupled beampattern synthesis with subarray-based frequency diverse array. Digit. Signal Process. 64, 49-59 (2017). https://doi.org/10.1016/j.dsp.2017.02.005

132. S. Saeed, I.M. Qureshi, W. Khan, A. Salman, Tangent hyperbolic circular frequency diverse array radars. J. Eng. 2016(3), 23-28 (2016). https://doi.org/10.1049/joe.2015.0194

133. B. Chen, X. Chen, Y. Huang, J. Guan, Transmit beampattern synthesis for the FDA radar. IEEE Antennas Wirel. Propag. Lett. 17(1), 98-101 (2018). https://doi.org/10.1109/LAWP.2017.2776957

134. K. Chen, S. Yang, Y. Chen, S. Qu, Accurate models of time-invariant beampatterns for frequency diverse arrays. IEEE Trans. Antennas Propag. 67(5), 3022-3029 (2019). https://doi.org/10.1109/TAP.2019.2896712

135. Z. Wang, Y. Song, T. Mu, Z. Ahmad, A short-range range-angle dependent beampattern synthesis by frequency diverse array. IEEE Access. 6, 22664-22669 (2018). https://doi.org/10.1109/ACCESS.2018.2827079

136. M. Tan, C. Wang, Z. Li, Correction analysis of frequency diverse array radar about time. IEEE Trans. Antennas Propag. 69(2), 834-847 (2021). https://doi.org/10.1109/TAP.2020.3016508

137. F. Mahdi, On the time-range dependency of the beampatterns produced by arbitrary antenna arrays: discussions on the misplaced expectations from frequency diverse arrays. arXiv:1903.03508v1 [physics.class-ph], pp. 1-5 (2019)

138. M. Fartookzadeh, Comments on "Frequency diverse array antenna using time-modulated optimized frequency offset to obtain time-invariant spatial fine focusing beampattern." IEEE Trans. Antennas Propag. 68(2), 1211-1212 (2020). https://doi.org/10.1109/TAP.2019.2955155

139. Z. Wang, Y. Song, T. Mu, Transmitted beampattern synthesis with waveform diverse arrays based on arctangent function. in IEEE 10th Sensor Array and Multichannel Signal Processing Workshop (SAM). Sheffield, UK 2018, 494-498 (2018). https://doi.org/10.1109/SAM.2018.8448526

140. Z. Ahmad, Z. Shi, C. Zhou, Y. Gu, Time-variant focused range-angle dependent beampattern synthesis by frequency diverse array radar. IET Signal Process. 14(6), 352-360 (2020). https://doi.org/10.1049/iet-spr.2020.0021

141. S.Y. Nusenu, A. Basit, Frequency-modulated diverse array transmit beamforming with bat metaheuristic optimisation. IET Radar Sonar Navig. 14(9), 1338-1342 (2020). https://doi.org/10.1049/iet-rsn.2019.0629

142. C. Cui, W. Li, X. Ye, Y. Hei, X. Shi, A short-time range-angle-decoupled beam pattern synthesis for frequency diverse arrays. IET Microw. Antennas Propag. 1-16 (2021). https://doi.org/10.1049/mia2.12099 
143. A. Basit, W.-Q. Wang, S.Y. Nusenu, Adaptive transmit array sidelobe control using FDA-MIMO for tracking in joint radar-communications. Digit. Signal Process. 97, 102619 (2020). https://doi.org/10.1016/j.dsp.2019.102619

144. X. Wu, H. Shao, J. Lin, Q. Li, Q. Shi, High-speed user-centric beampattern synthesis via frequency diverse array. IEEE Trans. Signal Process. 69, 1226-1241 (2021). https://doi.org/10.1109/TSP.2021.3054988

145. Z. Ahmad, Z. Shi, C. Zhou, Time-variant focusing range-angle dependent beampattern synthesis by uniform circular frequency diverse array radar. IET Radar Sonar Navig. 15(1), 62-74 (2021). https://doi.org/10.1049/rsn2.12014

146. W.T. Li, C. Cui, X.T. Ye, X.W. Shi, H.C. So, Quasi-time-invariant 3-D focusing beampattern synthesis for conformal frequency diverse array. IEEE Trans. Antennas Propag. 68(4), 2684-2697 (2020). https://doi.org/10.1109/TAP.2019. 2955199

147. J. Xie, B. Qiu, Q. Wang, J. Qu, Broadcasting directional modulation based on random frequency diverse array. Wireless Commun. Mobile Comput. 2019, 5051490 (2019). https://doi.org/10.1155/2019/5051490

148. M.P. Daly, E.L. Daly, J.T. Bernhard, Demonstration of directional modulation using a phased array. IEEE Trans. Antennas Propag. 58(5), 1545-1550 (2010). https://doi.org/10.1109/TAP.2010.2044357

149. M.P. Daly, J.T. Bernhard, Directional modulation technique for phased arrays. IEEE Trans. Antennas Propag. 57(9), 2633-2640 (2009). https://doi.org/10.1109/TAP.2009.2027047

150. W.-Q. Wang, DM using FDA antenna for secure transmission. IET Microw. Antennas Propag. 11(3), 336-345 (2017). https://doi.org/10.1049/iet-map.2016.0303

151. J. Xiong, S.Y. Nusenu, W.-Q. Wang, Directional modulation using frequency diverse array for secure communications. Wirel. Pers. Commun. 95(3), 2679-2689 (2017). https://doi.org/10.1007/s11277-017-3949-1

152. Q. Cheng, J. Zhu, T. Xie, J. Luo, Z. Xu, Time-invariant angle-range dependent directional modulation based on timemodulated frequency diverse arrays. IEEE Access. 5, 26279-26290 (2017). https://doi.org/10.1109/ACCESS.2017. 2772246

153. J. Hu, S. Yan, F. Shu, J. Wang, J. Li, Y. Zhang, Artificial-noise-aided secure transmission with directional modulation based on random frequency diverse arrays. IEEE Access. 5, 1658-1667 (2017). https://doi.org/10.1109/ACCESS. 2017.2653182

154. B. Qiu, J. Xie, L. Wang, Y.Wang, Artificial-noise-aided secure transmission for proximal legitimate user and eavesdropper based on frequency diverse arrays. IEEE Access. 6, 52531-52543 (2018). https://doi.org/10.1109/ACCESS. 2018.2869529

155. J. Lin, Q. Li, J. Yang, H. Shao, W.-Q. Wang, Physical-layer security for proximal legitimate user and eavesdropper: a frequency diverse array beamforming approach. IEEE Trans. Inf. Forensics Security. 13(3), 671-684 (2018). https:// doi.org/10.1109/TIFS.2017.2765500

156. B. Qiu, M. Tao, L. Wang, J. Xie, Y. Wang, Multi-beam directional modulation synthesis scheme based on frequency diverse array. IEEE Trans. Inf. Forensics Secur. 14(10), 2593-2606 (2019). https://doi.org/10.1109/TIFS.2019.2900942

157. T. Xie, J. Zhu, Y. Li, Artificial-noise-aided zero-forcing synthesis approach for secure multi-beam directional modulation. IEEE Commun. Lett. 22(2), 276-279 (2018). https://doi.org/10.1109/LCOMM.2017.2772228

158. B. Qiu, L. Wang, J. Xie, Z. Zhang, Y. Wang, M. Tao, Multi-beam index modulation with cooperative legitimate users schemes based on frequency diverse array. IEEE Trans. Veh. Technol. 69(10), 11028-11041 (2020). https://doi.org/ 10.1109/TVT.2020.3007003

159. Q. Cheng, V. Fusco, J. Zhu, S. Wang, C. Gu, SVD-aided multi-beam directional modulation scheme based on frequency diverse array. IEEE Wireless Commun. Lett. 9(3), 420-423 (2020). https://doi.org/10.1109/LWC.2019.29604 93

160. S.Y. Nusenu, Authentication and secrecy of multicast communication scenario: artificial noise-aided Costas sequence matrix FDA approach. Secur. Commun. Netw. 2020, 2194840 (2020). https://doi.org/10.1155/2020/ 2194840

161. S. Ke, M. He, X. Bu, W. Cai, A leakage-based directional modulation scheme for frequency diverse array in robot swarm networks. IEEE Access. 8, 107823-107837 (2020). https://doi.org/10.1109/ACCESS.2020.2998938

162. S. Ke, J. An, and S. Wang, Synthesis-free directional modulation for retrodirective frequency diverse array. Sci. China Inf. Sci. 63, 202304 (20208). https://doi.org/10.1007/s11432-020-2853-9.

163. S. Wang, S. Yan, J. Zhang, N. Yang, R. Chen, F. Shu, Secrecy zone achieved by directional modulation with random frequency diverse array. IEEE Trans. Veh. Technol. 70(2), 2001-2006 (2021). https://doi.org/10.1109/TVT.2021.30548 03

164. Q. Cheng, S. Wang, V. Fusco, F. Wang, J. Zhu, C. Gu, Physical-layer security for frequency diverse array-based directional modulation in fluctuating two-ray fading channels. IEEE Trans. Wireless Commun. 20(7), 4190-4204 (2021). https://doi.org/10.1109/TWC.2021.3056521

165. G. Babur, P. Aubry, F. Chevalier, Simple transmit diversity technique for phased array radar. IET Radar Sonar Navig. 10(6), 1046-1056 (2016). https://doi.org/10.1049/iet-rsn.2015.0311

\section{Publisher's Note}

Springer Nature remains neutral with regard to jurisdictional claims in published maps and institutional affiliations. 